Software Engineering

$\int$ SEN Software ENgineering

Symmetries in jump-diffusion models with applications in option pricing and credit risk

J.K. Hoogland, C.D.D. Neumann, M.H. Vellekoop

RePORT SEN-R0202 JanUaRY 31, 2002 
CWI is the National Research Institute for Mathematics and Computer Science. It is sponsored by the Netherlands Organization for Scientific Research (NWO).

$\mathrm{CWI}$ is a founding member of ERCIM, the European Research Consortium for Informatics and Mathematics.

CWI's research has a theme-oriented structure and is grouped into four clusters. Listed below are the names of the clusters and in parentheses their acronyms.

Probability, Networks and Algorithms (PNA)

\section{Software Engineering (SEN)}

Modelling, Analysis and Simulation (MAS)

Information Systems (INS)

Copyright @ 2001, Stichting Centrum voor Wiskunde en Informatica

P.O. Box 94079, 1090 GB Amsterdam (NL)

Kruislaan 413, 1098 SJ Amsterdam (NL)

Telephone +31205929333

Telefax +31 205924199 


\title{
Symmetries in Jump-Diffusion Models with Applications in Option Pricing and Credit Risk
}

\author{
J.K. Hoogland \& C.D.D. Neumann \\ CWI, P.O. Box 94079 \\ 1090 GB Amsterdam \\ The Netherlands \\ jiri@cwi.nl, neumann@cwi.nl \\ M.H. Vellekoop \\ Department of Applied Mathematics \\ University of Twente \\ P.O. Box 217, 7500 AE Enschede \\ The Netherlands \\ m.h.vellekoop@math.utwente.nl
}

\begin{abstract}
It is a well known fact that local scale invariance plays a fundamental role in the theory of derivative pricing. Specific applications of this principle have been used quite often under the name of 'change of numeraire', but in recent work it was shown that when invoked as a fundamental first principle, it provides a powerful alternative method for the derivation of prices and hedges of derivative securities, when prices of the underlying tradables are driven by Wiener processes. In this article we extend this work to the pricing problem in markets driven not only by Wiener processes but also by Poisson processes, i.e. jump-diffusion models. It is shown that in this case too, the focus on symmetry aspects of the problem leads to important simplifications of, and a deeper insight into the problem. Among the applications of the theory we consider the pricing of stock options in the presence of jumps, and Lévy-processes. Next we show how the same theory, by restricting the number of jumps, can be used to model credit risk, leading to a 'market model' of credit risk. Both the traditional Duffie-Singleton and Jarrow-Turnbull models can be described within this framework, but also more general models, which incorporate default correlation in a consistent way. As an application of this theory we look at the pricing of a credit default swap (CDS) and a first-to-default basket option.
\end{abstract}

2000 Mathematics Subject Classification: 35R10, 58J35, 58J70, 60H10, 91B24

Keywords and Phrases: option pricing, jump diffusion, local scale invariance, homogeneity, partial differential difference equations

Note: Work carried out under theme SEN4 "Evolutionary Systems and Applied Algorithmics"

\section{INTRODUCTION}

In the last thirty years, a considerable amount of scientific effort has been put into attempts to generalize the highly successful derivative pricing methods of Black and Scholes [BS73] to so called jump-diffusion models. In these models, the Wiener processes which were used by Black and Scholes to model stock dynamics, are complemented by Poisson processes which cause unprevisible discontinuities in the stock price [Mer76]. Such discontinuities are needed to incorporate unexpected sudden market events in price models. Possible applications are the modeling of stock market crashes, interest rates and credit risk. In general, the addition of Poisson processes causes the market model to be incomplete, since the extra stochastic factors cannot be offset by existing contracts, and this makes perfect replication of derivatives impossible. 
The traditional way to circumvent this problem is to use standard no-arbitrage pricing theory, in which prices are calculated as an expectation under some equivalent martingale measure. It is well known that for an arbitrage free but incomplete market such a measure exists but is not unique [HP81].

As an example consider the class of intensity based (or reduced form) credit risk models, such as Jarrow-Turnbull [JT95] and Duffie-Singleton [DS95]. In these models a martingale measure is postulated using an implied risk free default intensity, which is then fitted to market prices of e.g. corporate bonds. This approach has some marked disadvantages. For example, the relation between the real world measure and the equivalent martingale measure is not clear. Furthermore, it is not obvious what hedge ratios should be used (certainly not the usual Black Scholes deltas).

An alternative approach to the problem is to assume that the market, if not already complete, can be made complete by the introduction of additional contracts. The non-uniqueness in the pricing problem then translates into the freedom that one has in the specification of these new contracts. After completion of the market, prices and hedging strategies can be determined uniquely [BH99, VBH99, JM95, Jen99]. The question remains under what circumstances a market can be made complete. In Ref. [VBH99], for example, it was shown that if, for a given asset, the stochastic process which causes a jump is the only factor in the dynamics which is idiosyncratic and the percentage of loss upon a jump in the price is fixed and known a priori, it is possible to complete the market by the introduction of an insurance contract which pays a certain amount of money once a jump has occurred in exchange for an insurance premium during the lifetime of the contract.

In this article, we follow the alternative 'complete market' approach, making use of the tradable formalism as introduced in Refs. [HN99a, HN99b], thereby extending the powerful symmetry techniques to the realm of jump-diffusion models. We start out with a model which is complete. The core of the formalism is the idea that pricing problems should be formulated only in terms of self-financing objects, which we call tradables. From simple dimensional analysis one then finds that derivative prices must be homogeneous functions of degree one in these tradables. The consequent use of these proper coordinates makes numeraire invariance manifest, and the pricing problem more transparent. We find

- Pricing equations in the form of partial differential difference equations (PDDE's) for which the numeraire invariance of their solutions is manifest

- Explicit expressions for hedge ratios

- Analytic solutions for European style contracts in a certain class of jump-diffusion models

The relation between our approach and the martingale approach is illustrated, leading to a direct relation between real world and martingale measures. It is shown that the usual notion of a 'market price of risk' is not a numeraire invariant quantity for Poisson processes, in contrast to the case of Wiener processes. Therefore it is only well-defined with respect to an arbitrary choice of a tradable which is declared to be 'risk free' (usually the treasury bond).

As was mentioned before, the model, although derived under the assumption of market completeness, can deal with incomplete markets too, in as far as these markets can be made complete by the introduction of additional contracts for each independent source of jump risk. The freedom that we have in the specification of these contracts allows to introduce a specific market price of jump risk in the model. We illustrate this by considering the pricing of a derivative depending on a stock and a bond, where the stock is driven by one Wiener and several Poisson processes, taking the bond as numeraire. In a limit, this indicates how to price such contracts if the stock is driven by a Lévy-process.

By restricting the number of possible jumps of the individual Poisson processes to one, the very same theory can be applied to credit risk modeling. This approach could be called a 'market model' approach to credit risk, in the sense that we directly model the real world dynamics of the underlyings. Although conceptually different, the approach is closest to the intensity based approach and in fact 
we show that it is able to unify the models of Jarrow-Turnbull and Duffie-Singleton in one framework. It also allows the incorporation of default correlation in a straightforward and consistent way.

The outline of this article is as follows. In section 2 we refresh the basic ideas behind the tradable formalism. In section 3 we rederive pricing equations for pure diffusion models. Section 4 is the theoretic core of the article. It contains a general theory for pricing in jump-diffusion models, and investigates its symmetry properties. Some applications are given. In section 5, we look at consequences for pricing if Poisson processes are restricted to one jump only, having credit risk in mind. We review the Duffie-Singleton and Jarrow-Turnbull approaches and show that they both fit in the model and lead to almost identical pricing equations. As an application we calculate the value of a credit default swap in the two models. Next we look at multiple asset credit risk models, firstly considering the pricing of a first-to-default basket option, secondly deriving a consistent model with default correlation.

\section{EXPLOITING SYMMETRIES}

Any system of prices should be invariant under appropriate scaling transformations. Indeed, since the value of assets can only be defined in terms of the values of other assets, an economy-wide uniform proportional change of the values of all possible assets would simply amount to the introduction of a new measurement unit for value, and leave the economy essentially unchanged. Specific applications of this principle have been used quite often in finance under the name of 'change of numeraire' [GKR95], but in the recent article [HN99a] it was shown that when invoked as an overall fundamental first principle, it provides a powerful alternative method for the derivation of prices and hedges for derivative securities on tradable assets.

We assume a market in which $N+1$ tradables exist, and we denote the price at time $t$ of asset $i$ by $X_{t}^{i}$ with $i=0, \ldots, N$ and $\mathbf{X}_{t} \equiv\left(X_{t}^{0}, \ldots, X_{t}^{N}\right)$. These prices (in whatever a priori fixed numeraire we would like to choose) are modeled as stochastic processes $\left\{X_{t}^{i}, t \geq 0\right\}$ on a filtered probability space $\left(\Omega, \mathcal{F},\left\{\mathcal{F}_{t}\right\}_{t \geq 0}, P\right)$ where the filtration satisfies the usual assumptions. On this probability space we define canonical (possibly multi-dimensional) Wiener and Poisson Processes in the usual manner, which together generate the filtration $\left\{\mathcal{F}_{t}\right\}_{t \geq 0}$.

In this market we now consider the price of a European style derivative security $V$ which pays an amount $V(\mathbf{x}, T)$ at time of maturity $T$ only depending on the value of the price vector $\mathbf{x} \equiv$ $\left(x_{0}, x_{1}, \ldots, x_{N}\right)$ of the tradables at maturity $\mathbf{X}_{T}$. We assume that the market is complete, i.e. the price $V$ of the security at an earlier time $t \leq T$ exists and is uniquely determined by the market, and furthermore that this price is a function of the prices $X_{t}^{i}$ at time $t$ only. Under these assumptions we may write the price of the derivative security as $V(\mathbf{x}, t)$ and then a dimensional analysis argument shows that, since the prices of tradables and the security $V$ must be expressed in the same measurement units, we must have

$$
V\left(\mathbf{X}_{t}, t\right)=\sum_{i=0}^{N} \phi_{i}\left(\mathbf{X}_{t}, t\right) X_{t}^{i}
$$

for certain dimensionless quantities $\phi_{i}\left(\mathbf{X}_{t}, t\right)$, i.e. homogeneous functions of degree zero in $\mathbf{X}_{t}$. Consequently, the price function is homogeneous of degree 1

$$
V(\alpha \mathbf{x}, t)=\alpha V(\mathbf{x}, t)
$$

Differentiating with respect to $\alpha$ and setting $\alpha$ equal to one leads to the Euler formula

$$
V(\mathbf{x}, t)=\sum_{i=0}^{N} \frac{\partial V(\mathbf{x}, t)}{\partial x_{i}} x_{i}
$$

This simple formula is at the core of Black-Scholes pricing. Indeed, when prices are driven only by 
Wiener processes, we can prove that

$$
d V\left(\mathbf{X}_{t}, t\right)=\sum_{i=0}^{N} \frac{\partial V(\mathbf{x}, t)}{\partial x_{i}} d X_{t}^{i}
$$

given that $V$ satisfies a PDE (see next section). This shows that a portfolio consisting of an amount $\phi_{i}(\mathbf{x}, t)=\frac{\partial V(\mathbf{x}, t)}{\partial x_{i}}$ in each tradable $i$ not only replicates the security $V$, but is also self-financing, i.e. all changes in the price of the portfolio are caused by changes in the prices of the underlying tradables themselves. No money is put in or extracted from the portfolio.

When prices are driven by both Wiener and Poisson processes, the hedge ratios $\phi_{i}(\mathbf{x}, t)$ turn out to be more complicated, in general containing not only ordinary but also discrete derivatives of the price, and a little more work is required to find them. Nevertheless, the scale invariance expressed by Eqs. (2.1) and (2.2) still plays a fundamental role, as will be seen repeatedly in section 4.

\section{BlaCK-SCHOLES DYNAMICS}

To illustrate the ideas outlined in the previous section, consider the case of $N+1$ tradables with price processes $X_{t}^{i},(i=0, \ldots, N)$ driven by $N$ Wiener processes

$$
\frac{d X_{t}^{i}}{X_{t}^{i}}=\mu_{i}\left(\mathbf{X}_{t}, t\right) d t+\sum_{k=1}^{N} \sigma_{i}^{k}\left(\mathbf{X}_{t}, t\right) d W_{t}^{k}
$$

Here $\left\{\left(W_{t}, \mathcal{F}_{t}\right), t \geq 0\right\}$ is a standard $N$-dimensional Wiener process. The vector functions $\mu_{i}: \mathbf{R}^{N+1} \times$ $\mathbf{R} \rightarrow \mathbf{R}$ and $\sigma_{i}: \mathbf{R}^{N+1} \times \mathbf{R} \rightarrow \mathbf{R}$ are assumed to satisfy the standard growth conditions which guarantee uniqueness and existence of the solutions of this stochastic differential equation. Notice that the functions $\mu_{i}$ and $\sigma_{i}$ should all be homogeneous functions of degree 0 . In other words, they can be functions of ratios $X_{t}^{i} / X_{t}^{j}$ of tradable prices only, since the substituted values have to be dimensionless. For these dynamics we have by Itô's rule that

$$
d V\left(\mathbf{X}_{t}, t\right)=\mathcal{L} V\left(\mathbf{X}_{t}, t\right) d t+\sum_{i=0}^{N} \frac{\partial V\left(\mathbf{X}_{t}, t\right)}{\partial x_{i}} d X_{t}^{i}
$$

with

$$
\mathcal{L} V(\mathbf{x}, t) \equiv \frac{\partial V(\mathbf{x}, t)}{\partial t}+\frac{1}{2} \sum_{i, j=0}^{N} \sum_{k=1}^{N} \sigma_{i}^{k}(\mathbf{x}, t) \sigma_{j}^{k}(\mathbf{x}, t) x_{i} x_{j} \frac{\partial^{2} V(\mathbf{x}, t)}{\partial x_{i} \partial x_{j}}
$$

and solving the partial differential equation $\mathcal{L} V(\mathbf{x}, t)=0$, subject to contract specific boundary conditions, then leads to a self-financing portfolio in terms of tradables, as we have seen in the previous section $^{1}$. This leads to a unique price for European style derivative securities before maturity $T$ by specifying the payoff $V(\mathbf{x}, T)$ as a boundary condition. Of course, other types of contracts can be priced too, either by modifying the boundary conditions (e.g. American type contracts) or, in the case of strong path-dependence, by introducing specific derived tradables, which make the problem Markovian again (e.g. Asians, see [HNO0a]). Note that the pricing PDE does not contain first order partial derivatives w.r.t. the tradables. This has important advantageous consequences when implementing numerical schemes to solve the equation, see Ref. [HN00b].

\footnotetext{
${ }^{1}$ Assuming that second order partial derivatives of the price function with respect to the tradables, and the first order derivative with respect to time exist.
} 


\subsection{Symmetries of the PDE}

The invariance of the price of a derivative security under a change of numeraire should be inherited by the pricing PDE. Of course, prices under a new numeraire can in general be expressed in terms of prices under the old numeraire as $\hat{\mathbf{X}}_{t} \equiv \mathbf{X}_{t} / Y_{t}$, where the process $Y_{t}$ which defines the numeraire change can be arbitrary and stochastic, as long as it is always positive. Now suppose that $Y_{t}$ satisfies

$$
\frac{d Y_{t}}{Y_{t}}=\nu\left(\mathbf{X}_{t}, t\right) d t+\sum_{k=1}^{N} g_{k}\left(\mathbf{X}_{t}, t\right) d W_{t}^{k}
$$

Consistency requires that $\nu$ and $g_{k}$ are homogeneous functions of degree 0 in $\mathbf{X}_{t}$, so that $\nu\left(\mathbf{X}_{t}, t\right)=$ $\nu\left(\hat{\mathbf{X}}_{t}, t\right)$ and similar for $g_{k}$. These functions should also satisfy the standard growth conditions. By using Itô's rule, we find that the prices under the new numeraire satisfy

$$
\begin{aligned}
\frac{d \hat{X}_{t}^{i}}{\hat{X}_{t}^{i}}= & \left(\mu_{i}\left(\hat{\mathbf{X}}_{t}, t\right)-\nu\left(\hat{\mathbf{X}}_{t}, t\right)-g_{k}\left(\hat{\mathbf{X}}_{t}, t\right)\left(\sigma_{i}^{k}\left(\hat{\mathbf{X}}_{t}, t\right)-g_{k}\left(\hat{\mathbf{X}}_{t}, t\right)\right)\right) d t \\
& +\sum_{k=1}^{N}\left(\sigma_{i}^{k}\left(\hat{\mathbf{X}}_{t}, t\right)-g_{k}\left(\hat{\mathbf{X}}_{t}, t\right)\right) d W_{t}^{k}
\end{aligned}
$$

If we write down the pricing PDE in terms of the rescaled tradables, we find extra terms, proportional to the $g_{k}(\hat{\mathbf{x}}, t)$ :

$$
\begin{aligned}
& \mathcal{L} V(\hat{\mathbf{x}}, t)=\frac{\partial V(\hat{\mathbf{x}}, t)}{\partial t} \\
& \quad+\frac{1}{2} \sum_{i, j=0}^{N} \sum_{k=1}^{N}\left(\sigma_{i}^{k}(\hat{\mathbf{x}}, t)-g_{k}(\hat{\mathbf{x}}, t)\right)\left(\sigma_{j}^{k}(\hat{\mathbf{x}}, t)-g_{k}(\hat{\mathbf{x}}, t)\right) \hat{x}_{i} \hat{x}_{j} \frac{\partial^{2} V(\hat{\mathbf{x}}, t)}{\partial \hat{x}_{i} \partial \hat{x}_{j}}=0
\end{aligned}
$$

Now it is exactly the homogeneity property of $V$ which ensures that these new terms vanish. Indeed these terms can be written as

$$
\frac{1}{2} \sum_{k=1}^{N} \sum_{i=0}^{N} g_{k}(\hat{\mathbf{x}}, t)\left(g_{k}(\hat{\mathbf{x}}, t)-2 \sigma_{i}^{k}(\hat{\mathbf{x}}, t)\right) \hat{x}_{i}\left(\sum_{j=0}^{N} \hat{x}_{j} \frac{\partial^{2} V(\hat{\mathbf{x}}, t)}{\partial \hat{x}_{i} \partial \hat{x}_{j}}\right)
$$

and, by differentiating Eq. (2.2) once more, we find that for all $i$

$$
\sum_{j=0}^{N} \hat{x}_{j} \frac{\partial^{2} V(\hat{\mathbf{x}}, t)}{\partial \hat{x}_{i} \partial \hat{x}_{j}}=0
$$

We see that the solutions of the PDE for the price of a derivative security are invariant under changes of the numeraire. Furthermore, the price function itself should be invariant under the substitutions

$$
\sigma_{i}^{k}(\mathbf{x}, t) \rightarrow \sigma_{i}^{k}(\mathbf{x}, t)-g_{k}(\mathbf{x}, t) \quad \text { for all } i
$$

A clever choice of numeraire can simplify the computation of derivative security prices significantly. Indeed, picking tradable 0 as numeraire is easily seen to be equivalent to the choice $g_{k}=\sigma_{0}^{k}$ and $\nu=\mu_{0}$. The price process of this tradable then becomes trivial $d \hat{\mathbf{X}}_{t}=0$ and the dimension of the PDE reduces by one. This technique was used in Refs. [HNO0a, HN00c] to derive compact PDE's for the pricing of arithmetic Asian and passport options.

How does this all relate to the usual BS-framework? In that case, the interest rate is constant $r$ and there is a fixed numeraire, namely money. One of the tradables, say $\mathbf{X}^{0}$, will be the riskless bond, satisfying $d \mathbf{X}^{0}=r \mathbf{X}^{0} d t$, and so $\mathbf{X}^{0} \sim e^{r t}$ in units of money. Now since this is a deterministic function, it can be eliminated from the PDE, Eq. (3.2), leading to the familiar Black Scholes PDE. The disadvantages are obvious: this PDE contains drift terms, which can lead to numerical problems when numerically solving it and the manifest numeraire invariance is lost. In contrast, in our formalism the interest rate would only appear indirectly in prices via the value of $\mathbf{X}^{0}$. 
3.2 A simple example

Consider two tradables with price processes $X_{t}^{0,1}$ driven by one Wiener process $W_{t}$ :

$$
\frac{d X_{t}^{i}}{X_{t}^{i}}=\mu_{i}\left(X_{t}^{0}, X_{t}^{1}, t\right) d t+\sigma_{i}\left(X_{t}^{0}, X_{t}^{1}, t\right) d W_{t}, \quad(i=0,1)
$$

A European security with payoff $f\left(x_{0}, x_{1}\right)$ at maturity $T$ will have value $V\left(x_{0}, x_{1}, t\right)$ at time $t$, and this value should satisfy the following PDE

$$
\frac{\partial V\left(x_{0}, x_{1}, t\right)}{\partial t}+\frac{1}{2} \sum_{i, j=0,1} \sigma_{i}\left(x_{0}, x_{1}, t\right) \sigma_{j}\left(x_{0}, x_{1}, t\right) x_{i} x_{j} \frac{\partial^{2} V\left(x_{0}, x_{1}, t\right)}{\partial x_{i} \partial x_{j}}=0
$$

To reduce the dimension of the PDE we pick tradable 0 as numeraire. Introducing $\tau \equiv T-t$, $y \equiv x_{1} / x_{0}, V\left(x_{0}, x_{1}, t\right) \equiv x_{0} v(y, \tau)$, the PDE simplifies to

$$
-\frac{\partial v(y, \tau)}{\partial \tau}+\frac{1}{2} \sigma(y, \tau)^{2} y^{2} \frac{\partial^{2} v(y, \tau)}{\partial^{2} y}=0
$$

where we define

$$
\sigma(y, \tau) \equiv \sigma_{1}\left(x_{0}, x_{1}, T-\tau\right)-\sigma_{0}\left(x_{0}, x_{1}, T-\tau\right)
$$

Here we use the homogeneity of degree zero of the volatility functions $\sigma_{i}$, which implies that these functions can only depend upon the ratio $x_{1} / x_{0}$, i.e. on $y$. The boundary condition becomes $v(y, 0)=$ $f(1, y)$.

\section{Defaultable dynamics}

In this section we extend the pure diffusion case by adding jump-processes, where jumpsizes of the tradables are functions of the tradables and time.

\subsection{A simple example}

Before treating the general case we give a simple example. Consider two tradables with price processes satisfying

$$
\frac{d X_{t}^{i}}{X_{t_{-}}^{i}}=\mu_{i} d t+\left(\alpha_{i}-1\right) d N_{t}, \quad(i=0,1)
$$

Here $N_{t}$ denotes a Poisson process with a strictly positive intensity, driving both price processes, and the $\mu_{i}$ and $\alpha_{i}$ are constants for $i=0,1$. Since we want our prices to remain strictly positive, the $\alpha_{i}$ should also be strictly positive. No-arbitrage imposes further restrictions on the parameters: in order that $X_{t}^{1} / X_{t}^{0}$ is not a strictly in- or decreasing process, we must have the inequality $\left(\mu_{1}-\mu_{0}\right) /\left(\alpha_{1}-\alpha_{0}\right)<$ 0 . We will come back to this point in Section 4.1. A derivative security price $V\left(\mathbf{X}_{t}, t\right)$, where $\mathbf{X}_{t} \equiv\left(X_{t}^{0}, X_{t}^{1}\right)$, having second order derivatives w.r.t. $X_{t}^{0}$ and $X_{t}^{1}$, and first order derivative w.r.t. $t$, then satisfies (see Section 4.2)

$$
\begin{aligned}
d V\left(\mathbf{X}_{t}, t\right)= & \left(\frac{\partial V\left(\mathbf{X}_{t_{-}}, t\right)}{\partial t}+\sum_{i=0,1} \mu_{i} X_{t_{-}}^{i} \frac{\partial V\left(\mathbf{X}_{t_{-}}, t\right)}{\partial x_{i}}\right) d t \\
& +\left(V\left(\boldsymbol{\alpha} \mathbf{X}_{t_{-}}, t\right)-V\left(\mathbf{X}_{t_{-}}, t\right)\right) d N_{t}
\end{aligned}
$$

Here we introduced the shorthand notation $\boldsymbol{\alpha} \mathbf{X}_{t} \equiv\left(\alpha_{0} X_{t}^{0}, \alpha_{1} X_{t}^{1}\right)$. We can rewrite Eq. (4.1) now in such a way that we get terms proportional to the $d X_{t}^{i}$ and a remainder

$$
\begin{aligned}
d V\left(\mathbf{X}_{t}, t\right)= & \sum_{i=0,1} \phi_{i}\left(\mathbf{X}_{t_{-}}, t\right) d X_{t}^{i} \\
& +\left(\frac{\partial V\left(\mathbf{X}_{t_{-}}, t\right)}{\partial t}+\sum_{i=0,1} \mu_{i} X_{t_{-}}^{i}\left(\frac{\partial V\left(\mathbf{X}_{t_{-}}, t\right)}{\partial x_{i}}-\phi_{i}\left(\mathbf{X}_{t_{-}}, t\right)\right)\right) d t
\end{aligned}
$$


where we define

$$
\begin{aligned}
\phi_{0}(\mathbf{x}, t) & \equiv \frac{V\left(\alpha_{1} x_{0}, \alpha_{1} x_{1}, t\right)-V\left(\alpha_{0} x_{0}, \alpha_{1} x_{1}, t\right)}{x_{0}\left(\alpha_{1}-\alpha_{0}\right)} \\
\phi_{1}(\mathbf{x}, t) & \equiv \frac{V\left(\alpha_{0} x_{0}, \alpha_{0} x_{1}, t\right)-V\left(\alpha_{0} x_{0}, \alpha_{1} x_{1}, t\right)}{x_{1}\left(\alpha_{0}-\alpha_{1}\right)}
\end{aligned}
$$

such that

$$
x_{0} \phi_{0}(\mathbf{x}, t)+x_{1} \phi_{1}(\mathbf{x}, t)=V(\mathbf{x}, t)
$$

Here we use homogeneity to pull the $\alpha_{1}$ and $\alpha_{0}$ out of the $V$ 's in Eqs. (4.2) and (4.3) respectively. We see that the portfolio $V(x, t)$ is self-financing if the following partial differential difference equation (PDDE) holds.

$$
\mathcal{L} V(\mathbf{x}, t) \equiv \frac{\partial V(\mathbf{x}, t)}{\partial t}+\sum_{i=0,1} \mu_{i} x_{i}\left(\frac{\partial V(\mathbf{x}, t)}{\partial x_{i}}-\phi_{i}(\mathbf{x}, t)\right)=0
$$

Indeed, in that case we have the self-financing condition, by Eq. (4.4)

$$
d V\left(\mathbf{X}_{t}, t\right)=\sum_{i=0,1} \phi_{i}\left(\mathbf{X}_{t_{-}}, t\right) d X_{t}^{i}
$$

and we see that the $\phi_{i}(\mathbf{x}, t)$ are hedge ratios for the contract.

Symmetries of the PDDE Again, the PDDE should be invariant under a change of numeraire. So let us write $\hat{\mathbf{X}}_{t} \equiv \mathbf{X}_{t} / Y_{t}$ for prices under a new numeraire, where the process which defines the numeraire change satisfies

$$
\frac{d Y_{t}}{Y_{t_{-}}}=\kappa d t+(\gamma-1) d N_{t}
$$

The choice of $\kappa$ and $\gamma$ is arbitrary with the constraint that $\gamma>0$, since numeraires should be always positive. Then the price processes of the two tradables, expressed in terms of the new numeraire, become

$$
\frac{d \hat{X}_{t}^{i}}{\hat{X}_{t_{-}}^{i}}=\left(\mu_{i}-\kappa\right) d t+\left(\frac{\alpha_{i}}{\gamma}-1\right) d N_{t}
$$

Remember that homogeneity ensures that the functional form of $V$ is invariant under different numeraires, thus $V\left(X_{t}^{0}, X_{t}^{1}, t\right) \equiv Y V\left(\hat{X}_{t}^{0}, \hat{X}_{t}^{1}, t\right)$. How does the change of numeraire effect the hedge ratios, Eqs. (4.2) and (4.3)? The hedges are homogeneous of degree 0 in the tradables. In other words, they only depend on the ratio $\alpha_{1} / \alpha_{0}$ and therefore they are invariant under changes of numeraire, $\phi_{i}(\mathbf{x}, t)=\phi_{i}(\hat{\mathbf{x}}, t)$, as they should be. Now consider the PDDE, derived under the new numeraire

$$
\mathcal{L} V(\hat{\mathbf{x}}, t)=\frac{\partial V(\hat{\mathbf{x}}, t)}{\partial t}+\sum_{i=0,1}\left(\mu_{i}-\kappa\right) \hat{x}_{i}\left(\frac{\partial V(\hat{\mathbf{x}}, t)}{\partial \hat{x}_{i}}-\phi_{i}(\hat{\mathbf{x}}, t)\right)=0
$$

We see that it picks up extra terms, proportional to $\kappa$,

$$
\sum_{i=0,1} \hat{x}_{i}\left(\frac{\partial V(\hat{\mathbf{x}}, t)}{\partial \hat{x}_{i}}-\phi_{i}(\hat{\mathbf{x}}, t)\right)
$$

But these vanish because of homogeneity together with the replication condition Eq. (4.4) and so the PDDE is indeed invariant under changes of numeraire. Just as we could write down a PDE for the price of a derivative security in the pure diffusion case, it is possible to write down a PDDE for the pure jump case with fixed jump size, which is invariant under changes of numeraire. If we solve this PDDE, we find a price for the derivative security, depending on the two tradables with prices $X_{t}^{0}$ and $X_{t}^{1}$, together with the hedge ratios Eqs. (4.2) and (4.3). 
Solving the PDDE To solve Eq. (4.5) we introduce the notation $\tau \equiv T-t, y_{i} \equiv e^{-\mu_{i} \tau} x_{i}$, and $v(\mathbf{y}, \tau) \equiv V(\mathbf{x}, t)$. We can rewrite the PDDE as follows.

$$
-\frac{\partial v(\mathbf{y}, \tau)}{\partial \tau}-\frac{\mu_{0} \alpha_{1}-\mu_{1} \alpha_{0}}{\alpha_{1}-\alpha_{0}} v(\mathbf{y}, \tau)-\frac{\mu_{1}-\mu_{0}}{\alpha_{1}-\alpha_{0}} v(\boldsymbol{\alpha} \mathbf{y}, \tau)=0
$$

To get rid of the second term we multiply the PDDE with an integrating factor. This then leads to

$$
\frac{\partial f(\mathbf{y}, \tau)}{\partial \tau}=\lambda f(\boldsymbol{\alpha} \mathbf{y}, \tau)=f(\boldsymbol{\beta} \mathbf{y}, \tau)
$$

where $f(\mathbf{y}, \tau) \equiv v(\mathbf{y}, \tau) \exp \left(\frac{\mu_{0} \alpha_{1}-\mu_{1} \alpha_{0}}{\alpha_{1}-\alpha_{0}} \tau\right), \lambda \equiv-\frac{\mu_{1}-\mu_{0}}{\alpha_{1}-\alpha_{0}}$, and $\boldsymbol{\beta} \equiv \lambda \boldsymbol{\alpha}$. We used homogeneity to pull $\lambda$ inside the function $f$. As it will turn out, the $\boldsymbol{\beta}$ are the crucial parameters in the pricing problem. Using the ansatz

$$
f(\mathbf{y}, \tau)=\sum_{n \geq 0} w_{n}(\tau) g\left(\left(\beta_{0}\right)^{n} y_{0},\left(\beta_{1}\right)^{n} y_{1}\right)=\sum_{n \geq 0} g\left(w_{n}\left(\beta_{0} \tau\right) y_{0}, w_{n}\left(\beta_{1} \tau\right) y_{1}\right)
$$

it is easy to prove that Eq. (4.8), with $w_{n}(\tau)=\frac{\tau^{n}}{n !}$ and $g(\mathbf{y})=v(\mathbf{y}, 0)$, solves the PDDE in Eq. (4.7). Putting everything back together, the solution becomes

$$
V(\mathbf{x}, t)=\sum_{n \geq 0} V\left(x_{0} \frac{\left(\beta_{0} \tau\right)^{n}}{n !} e^{-\beta_{0} \tau}, x_{1} \frac{\left(\beta_{1} \tau\right)^{n}}{n !} e^{-\beta_{1} \tau}, T\right)
$$

Note that the parameters describing the dynamics only enter the solution via $\boldsymbol{\beta}$. These objects are numeraire invariant as can be seen easily by inspection, using $\mu_{i} \rightarrow \mu_{i}-\kappa, \alpha_{i} \rightarrow \alpha_{i} / \gamma$. Now it also becomes completely obvious that in order to avoid arbitrage, we have to restrict the $\boldsymbol{\beta}$ to be positive, or equivalently $\lambda>0$ since $\alpha_{i}>0$. Otherwise an option with positive payoff at maturity could have a negative value at an earlier time. In the present example, this leads to the following restriction, as we mentioned before

$$
\lambda=-\frac{\mu_{1}-\mu_{0}}{\alpha_{1}-\alpha_{0}}>0
$$

Finally note that there is no reference to the intensity of the real world process $N_{t}$. It completely drops out of the problem. This should indeed be the case since we have hedged our position and once a perfect hedge has been found which annihilates jump risk, we become indifferent to the frequency with which jumps occur. Note that this result still holds if the jump intensity in the real world measure would be a stochastic process. This does not mean that the real world intensity does not play any role at all in pricing. In practice, the jump intensity is closely related to the magnitude of the drift terms, which do enter the price formulas.

An interpretation Let us try to connect this result to the usual formulation in terms of riskneutral expectations. By using homogeneity, we can rewrite Eq. (4.9) as

$$
V(\mathbf{x}, t)=\sum_{n \geq 0} \frac{\left(\beta_{0} \tau\right)^{n}}{n !} e^{-\beta_{0} \tau} V\left(x_{0}, x_{1}\left(\frac{\beta_{1}}{\beta_{0}}\right)^{n} e^{-\left(\beta_{1}-\beta_{0}\right) \tau}, T\right)
$$

In this form, the formula can be interpreted as an expectation under a Poisson process with intensity $\beta_{0}$, where $x_{1}$ makes jumps of size $\beta_{1} / \beta_{0}=\alpha_{1} / \alpha_{0}$ and has a drift $-\left(\beta_{1}-\beta_{0}\right)$ relative to $x_{0}$. Now let's assume that $x_{0}$ is a 'risk-free' bond ${ }^{2}$. This corresponds to setting $\alpha_{0}=1$ and $\mu_{0}=r$. Further assume that $x_{1}$ is a risky bond, making downward jumps, so $\alpha_{1}=\alpha<1$ and $\mu_{1}=\mu$. Then

$$
\beta_{0}=\lambda \quad, \quad \beta_{1}=\lambda \alpha \quad, \quad \lambda=-\frac{\mu-r}{\alpha-1}
$$

\footnotetext{
${ }^{2}$ Of course, the notion of a 'risk-free' asset is defined relative to some numeraire, usually money, and means that under this numeraire the dynamics of that particular asset is deterministic.
} 
The no-arbitrage condition now amounts to $\mu>r$. This has a nice intuitive interpretation: any downward jumprisk should be compensated for by a higher return than the riskfree rate (this is known as the credit spread). In fact we can rewrite the definition of $\lambda$ as follows

$$
\mu=r+\lambda(1-\alpha) \geq r
$$

This suggest that $\lambda(1-\alpha)=\beta_{0}-\beta_{1}$ denotes the credit spread due to default risk and $\lambda$ could be interpreted as the 'market price of default risk', as was already noted in Ref. [VBH99]. One should however be careful to attach an invariant meaning to the latter, since it is not numeraire invariant. Indeed, if we change the numeraire to $x_{1}$, which corresponds to setting $\kappa=\mu$ and $\gamma=\alpha$ in Eq. (4.6), we find that $\lambda \rightarrow \alpha \lambda$. In this numeraire it would be more natural to rewrite Eq. (4.9) as follows

$$
V(\mathbf{x}, t)=\sum_{n \geq 0} \frac{\left(\beta_{1} \tau\right)^{n}}{n !} e^{-\beta_{1} \tau} V\left(x_{0}\left(\frac{\beta_{0}}{\beta_{1}}\right)^{n} e^{-\left(\beta_{0}-\beta_{1}\right) \tau}, x_{1}, T\right)
$$

But in this form, the formula can be interpreted as an expectation under a Poisson process with intensity $\beta_{1}$, where $x_{0}$ makes jumps of size $\beta_{0} / \beta_{1}=\alpha_{0} / \alpha_{1}=1 / \alpha>1$ and has a drift $-\left(\beta_{0}-\beta_{1}\right)$ relative to $x_{0}$. So we recover the well known fact that the choice of a martingale measure is also relative to the choice of a numeraire. Only the symmetric formula Eq. (4.9) is canonical and therefore, in our view, more fundamental.

\subsection{The general case}

Let us now consider the general case, where the price processes of the tradables are driven by both Wiener and Poisson processes. To this end we consider $N+1$ tradables with price processes $X_{t}^{i}(i=$ $0, \ldots, N)$ of the form

$$
\frac{d X_{t}^{i}}{X_{t_{-}}^{i}}=\mu_{i}\left(\mathbf{X}_{t_{-}}, t\right) d t+\sum_{m=1}^{M} \sigma_{i}^{m}\left(\mathbf{X}_{t_{-}}, t\right) d W_{t}^{m}+\sum_{r=1}^{R}\left(\alpha_{i}^{r}\left(\mathbf{X}_{t_{-}}, t\right)-1\right) d N_{t}^{r}
$$

where $\left\{\left(W_{t}, \mathcal{F}_{t}\right), t \geq 0\right\}$ and $\left\{\left(N_{t}, \mathcal{F}_{t}\right), t \geq 0\right\}$ are $M$ standard Wiener and $R$ Poisson processes with $M+R=N$, all independent of each other, and we assume that the Poisson processes all have a strictly positive intensity for all $t \geq 0$. We will again find that the actual values of the intensities do not play any role in the pricing equations. The functions $\mu_{i}(\mathbf{x}, t), \sigma_{i}^{m}(\mathbf{x}, i)$ and $\alpha_{i}^{r}(\mathbf{x}, t)$ are deterministic, known in advance and should be homogeneous of degree zero in the tradables. They will be restricted by no-arbitrage constraints. In order to keep the notation transparent, we will omit the parameters of these functions in what follows.

The first step: invoking Itô's rule We want to compute the price of a derivative security whose price $V\left(\mathbf{X}_{t}, t\right)$ depends on tradables satisfying Eq. (4.10). For any function $V(\mathbf{x}, t)$ for which the second order derivatives in $\mathbf{x}$ and the first order derivative in $t$ exist, we can write the generalized Itô rule:

$$
\begin{aligned}
& V\left(\mathbf{X}_{t}, t\right)-V\left(\mathbf{X}_{0}, 0\right)= \\
& =\int_{0}^{t} \frac{\partial V\left(\mathbf{X}_{s-}, s\right)}{\partial s} d s+\sum_{i=0}^{N} \int_{0}^{t} \frac{\partial V\left(\mathbf{X}_{s-}, s\right)}{\partial x_{i}} d X_{s}^{i} \\
& \quad+\frac{1}{2} \sum_{i, j=0}^{N} \int_{0}^{t} \frac{\partial^{2} V\left(\mathbf{X}_{s-}, s\right)}{\partial x_{i} \partial x_{j}} d\left[X^{i}, X^{j}\right]_{s}^{c} \\
& \quad+\sum_{0<s \leq t}\left(V\left(\mathbf{X}_{s}, s\right)-V\left(\mathbf{X}_{s-}, s\right)-\sum_{i=0}^{N} \frac{\partial V\left(\mathbf{X}_{s-}, s\right)}{\partial x_{i}} \Delta X_{s}^{i}\right)
\end{aligned}
$$


where $\left\{\left[X^{i}, X^{j}\right]_{t}^{c}, t \geq 0\right\}$ is the continuous part of the quadratic covariation process associated with the processes $X_{t}^{i}$ and $X_{t}^{j}$ and $\Delta X_{s}^{i} \equiv X_{s}^{i}-X_{s_{-}}^{i}$. Note that all functions only need to be evaluated on the left-continuous part of the process. In short-hand notation and by obvious substitutions this means that

$$
\begin{aligned}
& d V\left(\mathbf{X}_{t}, t\right)= \\
& =\sum_{i=0}^{N} X_{t_{-}}^{i} \frac{\partial V\left(\mathbf{X}_{t_{-}}, t\right)}{\partial x_{i}}\left(\mu_{i} d t+\sum_{m=1}^{M} \sigma_{i}^{m} d W_{t}^{m}+\sum_{r=1}^{R}\left(\alpha_{i}^{r}-1\right) d N_{t}^{r}\right) \\
& \quad+\left(\frac{\partial V\left(\mathbf{X}_{t_{-}}, t\right)}{\partial t}+\frac{1}{2} \sum_{i, j=0}^{N} \sum_{m=1}^{M} \sigma_{i}^{m} \sigma_{j}^{m} X_{t_{-}}^{i} X_{t_{-}}^{j} \frac{\partial^{2} V\left(\mathbf{X}_{t_{-}}, t\right)}{\partial x_{i} \partial x_{j}}\right) d t \\
& \quad+\sum_{r=1}^{R}\left(V\left(\boldsymbol{\alpha}^{r} \mathbf{X}_{t_{-}}, t\right)-V\left(\mathbf{X}_{t_{-}}, t\right)-\sum_{i=0}^{N}\left(\alpha_{i}^{r}-1\right) X_{t_{-}}^{i} \frac{\partial V\left(\mathbf{X}_{t_{-}}, t\right)}{\partial x_{i}}\right) d N_{t}^{r}
\end{aligned}
$$

where $\boldsymbol{\alpha}^{r} \mathbf{X}_{t_{-}} \equiv\left(\alpha_{0}^{r} X_{t_{-}}^{0}, \ldots, \alpha_{N}^{r} X_{t_{-}}^{N}\right)$. Now, just as in the previous cases, we want to use this equation to derive conditions under which a given portfolio is self-financing. To this end, we add terms of the form

$$
\phi_{i}\left(\mathbf{X}_{t_{-}}, t\right)\left(d X_{t}^{i}-\mu_{i} X_{t_{-}}^{i} d t+\sum_{m=1}^{M} \sigma_{i}^{m} X_{t_{-}}^{i} d W_{t}^{m}+\sum_{r=1}^{R}\left(\alpha_{i}^{r}-1\right) X_{t_{-}}^{i} d N_{t}^{r}\right)
$$

which vanish by virtue of the dynamic equations. The functions $\phi_{i}\left(\mathbf{X}_{t_{-}}, t\right)$ constitute hedge ratios which are yet to be determined. They must only depend on the left-continuous processes $\mathbf{X}_{t_{-}}$because we cannot allow our hedging strategy to anticipate the sudden jumps. The dynamic equation for $V$ now becomes

$$
\begin{aligned}
& d V\left(\mathbf{X}_{t}, t\right)= \\
&= \sum_{i=0}^{N} \phi_{i}\left(\mathbf{X}_{t_{-}}, t\right) d X_{t}^{i} \\
&+\left\{\frac{\partial V\left(\mathbf{X}_{t_{-}}, t\right)}{\partial t}+\frac{1}{2} \sum_{i, j=0}^{N} \sum_{m=1}^{M} \sigma_{i}^{m} \sigma_{j}^{m} X_{t_{-}}^{i} X_{t_{-}}^{j} \frac{\partial^{2} V\left(\mathbf{X}_{t_{-}}, t\right)}{\partial x_{i} \partial x_{j}}\right. \\
&\left.+\sum_{i=0}^{N} \mu_{i} X_{t_{-}}^{i}\left(\frac{\partial V\left(\mathbf{X}_{t_{-}}, t\right)}{\partial x_{i}}-\phi_{i}\left(\mathbf{X}_{t_{-}}, t\right)\right)\right\} d t \\
&+\sum_{m=1}^{M}\left\{\sum_{i=0}^{N} \sigma_{i}^{m} X_{t_{-}}^{i}\left(\frac{\partial V\left(\mathbf{X}_{t_{-}}, t\right)}{\partial x_{i}}-\phi_{i}\left(\mathbf{X}_{t_{-}}, t\right)\right)\right\} d W_{t}^{m} \\
&+\sum_{r=1}^{R}\left\{V\left(\alpha^{r} \mathbf{X}_{t_{-}}, t\right)-V\left(\mathbf{X}_{t_{-}}, t\right)\right. \\
&\left.\quad-\sum_{i=0}^{N}\left(\alpha_{i}^{r}-1\right) X_{t_{-}}^{i} \phi_{i}\left(\mathbf{X}_{t_{-}}, t\right)\right\} d N_{t}^{r} \\
& \stackrel{\text { def }}{=} \sum_{i=0}^{N} \phi_{i}\left(\mathbf{X}_{t_{-}}, t\right) d X_{t}^{i}+\mathcal{L} V\left(\mathbf{X}_{t_{-}}, t\right) d t \\
&+\sum_{m=1}^{M} \mathcal{L}_{W}^{m} V\left(\mathbf{X}_{t_{-}}, t\right) d W_{t}^{m}+\sum_{r=1}^{R} \mathcal{L}_{N}^{r} V\left(\mathbf{X}_{t_{-}}, t\right) d N_{t}^{r}
\end{aligned}
$$


where we defined

$$
\begin{aligned}
\mathcal{L} V(\mathbf{x}, t) \equiv & \frac{\partial V(\mathbf{x}, t)}{\partial t}+\frac{1}{2} \sum_{i, j=0}^{N} \sum_{m=1}^{M} \sigma_{i}^{m} \sigma_{j}^{m} x_{i} x_{j} \frac{\partial^{2} V(\mathbf{x}, t)}{\partial x_{i} \partial x_{j}} \\
& +\sum_{i=0}^{N} \mu_{i} x_{i}\left(\frac{\partial V(\mathbf{x}, t)}{\partial x_{i}}-\phi_{i}(\mathbf{x}, t)\right), \\
\mathcal{L}_{W}^{m} V(\mathbf{x}, t) \equiv & \sum_{i=0}^{N} \sigma_{i}^{m} x_{i}\left(\frac{\partial V(\mathbf{x}, t)}{\partial x_{i}}-\phi_{i}(\mathbf{x}, t)\right)
\end{aligned}
$$

for $m=1, \ldots, M$ and

$$
\mathcal{L}_{N}^{r} V(\mathbf{x}, t) \equiv V\left(\boldsymbol{\alpha}^{r} \mathbf{x}, t\right)-V(\mathbf{x}, t)-\sum_{i=0}^{N}\left(\alpha_{i}^{r}-1\right) x_{i} \phi_{i}(\mathbf{x}, t)
$$

for $r=1, \ldots, R$.

The self-financing portfolio conditions From Eq. (4.11) it is now obvious that a portfolio consisting of $\phi_{i}(x, t)$ assets $i$ at time $t$ will be self-financing if and only if

$$
\begin{array}{rlrl}
\mathcal{L} V(\mathbf{x}, t) & =0 & \\
\mathcal{L}_{W}^{m} V(\mathbf{x}, t) & =0 & & \forall m=1, \ldots, M \\
\mathcal{L}_{N}^{r} V(\mathbf{x}, t) & =0 & & \forall r=1, \ldots, R
\end{array}
$$

while at the same time,

$$
V(\mathbf{x}, t)=\sum_{i=0}^{N} \phi_{i}(\mathbf{x}, t) x_{i}
$$

Using homogeneity this last condition can be shown to be equivalent to

$$
\sum_{i=0}^{N} x_{i}\left(\frac{\partial V(\mathbf{x}, t)}{\partial x_{i}}-\phi_{i}(\mathbf{x}, t)\right)=0
$$

Note that it is easy to see that in the absence of jump processes, we must have $\phi_{i}(\mathbf{x}, t)=\frac{\partial V(\mathbf{x}, t)}{\partial x_{i}}$, i.e. we retrieve the usual Black-Scholes delta hedge. Now we use Eq. (4.15) and homogeneity to rewrite Eq. (4.14) in a slightly different form, which will turn out to be useful when solving the constraints

$$
\begin{aligned}
0= & \chi^{r}(\mathbf{x}, t) \\
= & V\left(\boldsymbol{\alpha}^{r} \mathbf{x}, t\right)-V(\mathbf{x}, t) \\
& +\sum_{i=0}^{N}\left(\alpha_{i}^{r}-1\right) x_{i}\left(\frac{\partial V(\mathbf{x}, t)}{\partial x_{i}}-\phi_{i}(\mathbf{x}, t)\right) \\
& +\sum_{i=0}^{N} x_{i} \frac{\partial V(\mathbf{x}, t)}{\partial x_{i}}-\sum_{i=0}^{N} \alpha_{i}^{r} x_{i} \frac{\partial V(\mathbf{x}, t)}{\partial x_{i}} \\
= & \sum_{i=0}^{N} \alpha_{i}^{r} x_{i}\left(\frac{\partial V(\mathbf{x}, t)}{\partial x_{i}}-\phi_{i}(\mathbf{x}, t)\right)-\sum_{i=0}^{N} \alpha_{i}^{r} x_{i} \frac{\partial V(\mathbf{x}, t)}{\partial x_{i}}+V\left(\boldsymbol{\alpha}^{r} \mathbf{x}, t\right)
\end{aligned}
$$


Indeed, collecting Eqs. (4.13),(4.15), and (4.16), we now have a set of $M+R+1=N+1$ linear equations for the $N+1$ unknown hedge ratios $\phi_{i}(x, t)$ :

$$
\left(\begin{array}{ccc}
1 & \cdots & 1 \\
\sigma_{0}^{1} & \cdots & \sigma_{N}^{1} \\
\vdots & \ddots & \vdots \\
\sigma_{0}^{M} & \cdots & \sigma_{N}^{M} \\
\alpha_{0}^{1} & \cdots & \alpha_{N}^{1} \\
\vdots & \ddots & \vdots \\
\alpha_{0}^{R} & \cdots & \alpha_{N}^{R}
\end{array}\right)\left(\begin{array}{c}
x_{0}\left(\frac{\partial V}{\partial x_{0}}-\phi_{0}\right) \\
\vdots \\
\vdots \\
\vdots \\
x_{N}\left(\frac{\partial V}{\partial x_{N}}-\phi_{N}\right)
\end{array}\right)=\left(\begin{array}{c}
0 \\
\vdots \\
0 \\
\sum_{i=0}^{N} \alpha_{i}^{1} x_{i} \frac{\partial V}{\partial x_{i}}-V\left(\boldsymbol{\alpha}^{1} \mathbf{x}, t\right) \\
\vdots \\
\sum_{i=0}^{N} \alpha_{i}^{R} x_{i} \frac{\partial V}{\partial x_{i}}-V\left(\boldsymbol{\alpha}^{R} \mathbf{x}, t\right)
\end{array}\right)
$$

Under obvious non-singularity conditions this has a unique solution. We will denote the matrix in the above equation by $A$. Substituting the solution in the remaining Eq. (4.12), we find

$$
\begin{aligned}
\mathcal{L} V(\mathbf{x}, t)= & \frac{\partial V(\mathbf{x}, t)}{\partial t}+\frac{1}{2} \sum_{i, j=0}^{N} \sum_{m=1}^{M} \sigma_{i}^{m} \sigma_{j}^{m} x_{i} x_{j} \frac{\partial^{2} V(\mathbf{x}, t)}{\partial x_{i} \partial x_{j}} \\
& +\sum_{i=0}^{N} \mu_{i} x_{i}\left(\frac{\partial V(\mathbf{x}, t)}{\partial x_{i}}-\phi_{i}(\mathbf{x}, t)\right) \\
= & \frac{\partial V(\mathbf{x}, t)}{\partial t}+\frac{1}{2} \sum_{i, j=0}^{N} \sum_{m=1}^{M} \sigma_{i}^{m} \sigma_{j}^{m} x_{i} x_{j} \frac{\partial^{2} V(\mathbf{x}, t)}{\partial x_{i} \partial x_{j}} \\
& +\sum_{r=1}^{R}\left(V\left(\boldsymbol{\beta}^{r} \mathbf{x}, t\right)-\sum_{i=0}^{N} \beta_{i}^{r} x_{i} \frac{\partial V(\mathbf{x}, t)}{\partial x_{i}}\right) .
\end{aligned}
$$

where we define quantities

$$
\beta_{i}^{r} \equiv \alpha_{i}^{r} \lambda_{r}, \quad \lambda_{r} \equiv-\sum_{j=0}^{N} \mu_{j} A_{j, M+r}^{-1}
$$

Again, we used homogeneity to pull the terms $\lambda_{r}$ inside the function $V$ using $V\left(\lambda_{r} \boldsymbol{\alpha}^{r} \mathbf{x}, t\right)=V\left(\boldsymbol{\beta}^{r} \mathbf{x}, t\right)$. At this point we have found the PDDE which the price of a derivative security must satisfy. It now remains to show that this PDDE respects the numeraire invariance. In fact, we will proceed to show that the $\beta_{i}^{r}$ are themselves numeraire invariant quantities, and (in Section 4.2) that the no-arbitrage condition requires them to be positive.

Symmetries of the PDDE By now, it should not surprise the reader that the pricing PDDE Eq. (4.18) respects numeraire invariance, since we explicitly used this fact in its derivation. Nevertheless we will once more prove this property, as a consistency check. In the present model, a general change of numeraire consists of:

- a shift of the drift-terms: $\mu_{i} \rightarrow \mu_{i}-\kappa$

- shifts of the volatility functions: $\sigma_{i}^{m} \rightarrow \sigma_{i}^{m}-g_{m}$

- the scaling of the jump sizes: $\alpha_{i}^{r} \rightarrow \alpha_{i}^{r} \gamma_{r}$

The proof of the invariance of the diffusion part of the PDDE is completely identical to the derivation in Section 3.1. The invariance of the jump part of the equation requires a little extra work. Let us consider Eq. (4.19) again

$$
\beta_{i}^{r} \equiv \alpha_{i}^{r} \lambda_{r}, \quad \lambda_{r} \equiv-\sum_{j=0}^{N} \mu_{j} A_{j, M+r}^{-1}
$$


The $\lambda_{r}$ can be viewed as part of a vector $\mathbf{v} \equiv\left(v_{0}, \varphi_{1}, \ldots, \varphi_{M},-\lambda_{1}, \ldots,-\lambda_{R}\right)$, which is a solution of

$$
\mu_{j}=\sum_{i=0}^{N} v_{i} A_{i j}=v_{0}+\sum_{m=1}^{M} \varphi_{m} \sigma_{j}^{m}-\sum_{r=1}^{R} \lambda_{r} \alpha_{j}^{r}
$$

Obviously, if we can prove the invariance of the $\boldsymbol{\beta}^{r}$, invariance of the PDDE follows. In fact we will show a little more, namely that the $\varphi_{m}$ are also numeraire invariant. First we consider the effect of scaling the jumpsizes $\alpha_{i}^{r} \rightarrow \alpha_{i}^{r} \gamma_{r}$. Then the matrix components $A_{i j}$ transform like

$$
A_{i j} \rightarrow D_{i} A_{i j}
$$

where $D \equiv\left(1, \ldots, 1, \gamma_{1}, \ldots, \gamma_{r}\right)$. This automatically implies that we should have $v_{i} \rightarrow D_{i}^{-1} v_{i}$ and thus $\lambda_{r} \rightarrow \lambda_{r} \gamma_{r}^{-1}$ and $\varphi_{m} \rightarrow \varphi_{m}$. Since $\beta_{i}^{r} \equiv \alpha_{i}^{r} \lambda_{r}$, we therefore have

$$
\beta_{i}^{r} \rightarrow\left(\alpha_{i}^{r} \gamma_{r}\right)\left(\lambda_{r} \gamma_{r}^{-1}\right)=\alpha_{i}^{r} \lambda_{r}=\beta_{i}^{r}
$$

Thus the $\boldsymbol{\beta}^{r}$ and $\varphi_{m}$ are invariant under scaling of the jump sizes. Now consider what happens when all $\mu_{j}$ are shifted by $-\kappa$. In this case we need a correction on $\mathbf{v}$ such that

$$
\mu_{j}-\kappa=\sum_{i=0}^{N}\left(v_{i}+\delta v_{i}\right) A_{i j}
$$

is satisfied. The particular form of $A$ shows that this can only be done by setting $\delta \mathbf{v}=(-\kappa, 0, \ldots, 0)$. But this shows that the $v_{i}$ with $i \neq 0$ are invariant under the shifts, and the same holds for $\boldsymbol{\beta}^{r}$ and $\varphi_{m}$. Note that we effectively use the portfolio replication condition Eq. (4.15). In a similar manner we can show the invariance under shifts $\sigma_{i}^{m} \rightarrow \sigma_{i}^{m}-g_{m}$ in the volatility functions. Indeed, we now need a correction such that

$$
\mu_{j}=\sum_{i=0}^{N}\left(v_{i}+\delta v_{i}\right) A_{i j}-\sum_{m=1}^{M}\left(v_{m}+\delta v_{m}\right) g_{m}
$$

is satisfied. Since the terms depending on $g_{m}$ do not depend on the index $j$, it is not hard to see that the proper choice is

$$
\delta \mathbf{v}=\left(\sum_{m=1}^{M} v_{m} g_{m}, 0, \ldots, 0\right)
$$

again showing the invariance of the $\boldsymbol{\beta}^{r}$ and $\varphi_{m}$ in this case.

Market prices of risk The 'market prices of risk' is a notion that is often used to indicate the willingness of the market to hold a risky asset compared to holding a risk-free asset. In the case of jump-diffusion more care should be taken when introducing such concepts. In particular we show that the usual definition of 'market-price of risk' for a Poisson process is not a numeraire invariant quantity. This is in contrast to the case of Wiener processes. Thus when talking about 'market price of risk' for a Poisson process, one should always make clear what particular numeraire is chosen. However, it is possible to formulate everything in terms of numeraire invariant quantities. This is exactly why we introduced the $\boldsymbol{\beta}^{r}$. Expressed in these objects no confusion can arise to what is meant. Let us once more consider Eq. (4.20)

$$
\mu_{i}=v_{0}+\sum_{m=1}^{M} \varphi_{m} \sigma_{i}^{m}-\sum_{r=1}^{R} \lambda_{r} \alpha_{i}^{r}
$$


As we have seen in the previous section, both left- and right-hand side are not numeraire invariant quantities. But it is straightforward to introduce a quantity which is numeraire invariant and tells us something about the 'market prices of risk'. Indeed, consider the difference $\mu_{i}-\mu_{j}$

$$
\mu_{i}-\mu_{j}=\sum_{m=1}^{M} \varphi_{m}\left(\sigma_{i}^{m}-\sigma_{j}^{m}\right)-\sum_{r=1}^{R} \lambda_{r}\left(\alpha_{i}^{r}-\alpha_{j}^{r}\right)
$$

This is the general, numeraire invariant, expression that provides a relation between the returns of the tradables, their volatilities, and jump sizes. The quantities that, in the literature, are called 'market prices of risk' are $\varphi_{m}$ and $\lambda_{r}$. Note however that only the $\varphi_{m}$ of these are numeraire invariant. The $\lambda_{r}$ are definitely not. This is a strong argument in favor of the $\beta_{i}^{r}=\alpha_{i}^{r} \lambda_{r}$ as the fundamental quantities for Poisson processes, since they are, like the $\varphi_{m}$, numeraire invariant. It is for this reason, that in the remainder of this article we will only use the $\beta_{i}^{r}$. Note that, in view of the discussion in subsection 4.1, the set $\left\{\beta_{i}^{1}, \ldots, \beta_{i}^{R}\right\}$ for a given $i$ has an interpretation as risk neutral intensities for the individual Poisson processes under the numeraire $X_{t}^{i}$.

The usual formulation The usual formulation of 'market price of risk' amounts to the choice of money as a numeraire. Next we pick a tradable, say tradable 0 , that we call 'risk-free' with drift $r$, no volatility $\sigma_{0}^{m}=0$, and no jumps, $\alpha_{0}^{r}=1$. Then Eq. (4.21) reduces to (with $j=0$ and $i=1, \ldots, N$ )

$$
\mu_{i}=r+\sum_{m=1}^{M} \varphi_{m} \sigma_{i}^{m}-\sum_{r=1}^{R} \lambda_{r}\left(\alpha_{i}^{r}-1\right)
$$

For the two simplest cases we can then easily write down the corresponding 'market prices of risk'. For the case where $M=1, R=0$ this amounts to (dropping indices)

$$
\varphi=\frac{\mu-r}{\sigma}
$$

and in the case where $M=0, R=1$ we get

$$
\lambda=\frac{\mu-r}{1-\alpha}
$$

A general solution In this section we present a solution of the PDDE $\mathcal{L} V=0$, with $\mathcal{L} V$ as given in Eq. (4.18), for the case where the $\mu_{i}(t)$ and $\sigma_{i}^{m}(t)$ only depend on $t$ and the $\alpha_{i}^{r}$ are constant. We consider a European style derivative security, where a payoff $V(\mathbf{x}, T)$ is specified as boundary condition at maturity $T$. In appendix A we prove that the price of such a contract at time $t<T$ is given by

$$
\begin{aligned}
& V(\mathbf{x}, t)= \\
& \quad \sum_{\mathbf{n} \geq 0} \int_{\mathbf{R}^{M}} V\left(x_{0} \phi\left(\mathbf{z}-\boldsymbol{\theta}_{0}\right) \pi_{\mathbf{n}}\left(\boldsymbol{\rho}_{0}\right), \ldots, x_{N} \phi\left(\mathbf{z}-\boldsymbol{\theta}_{N}\right) \pi_{\mathbf{n}}\left(\boldsymbol{\rho}_{N}\right), T\right) d \mathbf{z}
\end{aligned}
$$

where $\mathbf{z} \equiv\left(z_{1}, \ldots, z_{M}\right), \mathbf{n} \equiv\left(n_{1}, \ldots, n_{R}\right)$, and $\mathbf{n} \geq 0 \equiv\left\{\mathbf{n} \mid n_{1} \geq 0, \ldots, n_{R} \geq 0\right\}$. The $N+1$ vectors $\boldsymbol{\theta}_{i} \equiv\left(\theta_{i}^{1}, \ldots, \theta_{i}^{M}\right)$ have dimension $M$. They are found from a singular value decomposition of the time-integrated covariance matrix (which has full rank, since $A$ is assumed to be invertible)

$$
\sum_{m=1}^{M} \theta_{i}^{m}(t) \theta_{j}^{m}(t) \equiv \int_{t}^{T} \sum_{m=1}^{M} \sigma_{i}^{m}(u) \sigma_{j}^{m}(u) d u
$$

The $N+1$ vectors $\boldsymbol{\rho}_{i} \equiv\left(\rho_{i}^{1}, \ldots, \rho_{i}^{R}\right)$ are defined by

$$
\rho_{i}^{r}(t) \equiv \int_{t}^{T} \beta_{i}^{r}(u) d u
$$


The functions $\phi(\mathbf{z})$ and $\pi_{\mathbf{n}}\left(\boldsymbol{\rho}_{i}\right)$ are defined by

$$
\begin{aligned}
\phi(\mathbf{z}) & \equiv \prod_{m=1}^{M} \frac{1}{\sqrt{2 \pi}} e^{-\frac{1}{2} z_{m}^{2}} \\
\pi_{\mathbf{n}}\left(\boldsymbol{\rho}_{i}\right) & \equiv \prod_{r=1}^{R}\left(\frac{\left(\rho_{i}^{r}\right)^{n_{r}}}{n_{r} !} e^{-\rho_{i}^{r}}\right)
\end{aligned}
$$

Note that the general solution Eq. (4.23) is in a symmetric, canonical form. It is form invariant under numeraire changes. Of course, one could relate this solution to an expectation under some equivalent martingale measure, by choosing a numeraire and using homogeneity to bring some functions out of $V$, as we have shown in Section 4.1. However, we prefer the symmetric form, since it makes the numeraire invariance manifest.

The single pure jump process revisited Let us reconsider the example of Section 4.1, which corresponds to the case $R=1, M=0$. The price processes for the two tradables are given by

$$
\frac{d X_{t}^{i}}{X_{t_{-}}^{i}}=\mu_{i} d t+\left(\alpha_{i}-1\right) d N_{t}, \quad(i=0,1)
$$

where the $\alpha_{i}$ and $\mu_{i}$ are constant and $\alpha_{i}>0$. The corresponding matrix $A$ of constraints is given by

$$
A=\left(\begin{array}{cc}
1 & 1 \\
\alpha_{0} & \alpha_{1}
\end{array}\right)
$$

For $A$ to be invertible we need to have $\alpha_{0} \neq \alpha_{1}$. The corresponding $\boldsymbol{\beta}$ are

$$
\beta_{0}=-\alpha_{0} \frac{\mu_{1}-\mu_{0}}{\alpha_{1}-\alpha_{0}} \quad, \quad \beta_{1}=-\alpha_{1} \frac{\mu_{1}-\mu_{0}}{\alpha_{1}-\alpha_{0}}
$$

as expected. The no-arbitrage conditions $\beta_{i}>0$ become $\mu_{0}<\mu_{1}$ iff $\alpha_{0}>\alpha_{1}$. Since the $\boldsymbol{\beta}$ are constant, we readily write down the price of a European contract at time $t$, given the payoff $V(\mathbf{x}, T)$ at maturity $T$, using Eq. (4.23)

$$
V(\mathbf{x}, t)=\sum_{n \geq 0} V\left(x_{0} \pi_{n}\left(\beta_{0} \tau\right), x_{1} \pi_{n}\left(\beta_{1} \tau\right), T\right)
$$

It is now a simple matter to determine the equations for the hedge ratios $\phi_{i}(\mathbf{x}, t)$ in terms of the two tradables,

$$
\left(\begin{array}{l}
x_{0}\left(\frac{\partial V}{\partial x_{0}}-\phi_{0}\right) \\
x_{1}\left(\frac{\partial V}{\partial x_{1}}-\phi_{1}\right)
\end{array}\right)=\left(\begin{array}{cc}
1 & 1 \\
\alpha_{0} & \alpha_{1}
\end{array}\right)^{-1}\left(\begin{array}{c}
0 \\
\sum_{i=0,1} \alpha_{i} x_{i} \frac{\partial V}{\partial x_{i}}-V(\boldsymbol{\alpha} \mathbf{x}, t)
\end{array}\right)
$$

Solving for $\phi_{i}(\mathbf{x}, t)$ we get

$$
\begin{aligned}
& \phi_{0}(\mathbf{x}, t)=\frac{\alpha_{1} \sum_{i=0,1} x_{i} \frac{\partial V(\mathbf{x}, t)}{\partial x_{i}}-V(\boldsymbol{\alpha} \mathbf{x}, t)}{x_{0}\left(\alpha_{1}-\alpha_{0}\right)}=\frac{\alpha_{1} V(\mathbf{x}, t)-V(\boldsymbol{\alpha} \mathbf{x}, t)}{x_{0}\left(\alpha_{1}-\alpha_{0}\right)} \\
& \phi_{1}(\mathbf{x}, t)=\frac{\alpha_{0} \sum_{i=0,1} x_{i} \frac{\partial V(\mathbf{x}, t)}{\partial x_{i}}-V(\boldsymbol{\alpha} \mathbf{x}, t)}{x_{1}\left(\alpha_{0}-\alpha_{1}\right)}=\frac{\alpha_{0} V(\mathbf{x}, t)-V(\boldsymbol{\alpha} \mathbf{x}, t)}{x_{1}\left(\alpha_{0}-\alpha_{1}\right)}
\end{aligned}
$$

where we have used homogeneity again. 
Power tradables In our approach we stress the importance of using proper coordinates to formulate pricing problems. We have shown that this leads to the use of tradables, or self-financing objects as coordinates. Depending on the type of pricing problem it may be useful to introduce additional tradables, derived from the initial ones, to simplify calculations. In Ref. [HN99b] we introduced socalled power-tradables to derive compact expressions for exotic options in a diffusion setting. We can do similar things in the case of jump-diffusion processes. To this end consider tradables with the following payoff at maturity $T$ :

$$
V\left(x_{0}, \ldots, x_{N}, T\right)=\prod_{i=0}^{N} x_{i}^{\eta_{i}}
$$

where we have to impose the constraint $\sum_{i=0}^{N} \eta_{i}=1$ in order to get a homogeneous function of degree one. Now it is a simple matter to compute the value of a tradable with this payoff at an earlier time in the context of Sec. 4.2. Working out Eq. (4.23) for this case we arrive at

$$
V\left(x_{0}, \ldots, x_{N}, t\right)=e^{\xi(t)} \prod_{i=0}^{N} x_{i}^{\eta_{i}}
$$

where

$$
\xi(t)=-\frac{1}{2} \sum_{m=1}^{M} \sum_{i<j} \eta_{i} \eta_{j}\left(\theta_{i}^{m}-\theta_{j}^{m}\right)^{2}+\sum_{r=1}^{R}\left(\prod_{i=0}^{N}\left(\rho_{i}^{r}\right)^{\eta_{i}}-\sum_{i=0}^{N} \eta_{i} \rho_{i}^{r}\right)
$$

By using the relations $x_{i} \frac{\partial V(\mathbf{x}, t)}{\partial x_{i}}=\eta_{i} V(\mathbf{x}, t)$ and $V\left(\boldsymbol{\alpha}^{r} \mathbf{x}, t\right)=\prod_{i=0}^{N}\left(\alpha_{i}^{r}\right)^{\eta_{i}} V(\mathbf{x}, t)$ we find from Eq. (4.17) that the hedge ratios should satisfy

$$
A\left(\begin{array}{c}
x_{0} \phi_{0} \\
\vdots \\
x_{N} \phi_{N}
\end{array}\right)=\left(\begin{array}{c}
1 \\
\psi^{1} \\
\vdots \\
\psi^{M} \\
\chi^{1} \\
\vdots \\
\chi^{R}
\end{array}\right) V(\mathbf{x}, t), \quad \psi^{m}=\sum_{i=0}^{N} \sigma_{i}^{m} \eta_{i}, \quad \chi^{r}=\prod_{i=0}^{N}\left(\alpha_{i}^{r}\right)^{\eta_{i}}
$$

From this we derive the dynamic equations for the power tradable

$$
\begin{aligned}
\frac{d V_{t}}{V_{t_{-}}} & =\sum_{i=0}^{N} \phi_{i} \frac{d X_{t}^{i}}{V_{t_{-}}} \\
& =\left(v_{0}+\sum_{m=1}^{M} \psi^{m} \varphi_{m}-\sum_{r=1}^{R} \chi^{r} \lambda_{r}\right) d t+\sum_{m=1}^{M} \psi^{m} d W_{t}^{m}+\sum_{r=1}^{R}\left(\chi^{r}-1\right) d N_{t}^{r}
\end{aligned}
$$

where $v_{0}, \varphi_{m}, \lambda_{r}$ were defined in Eq. (4.20). One important use of the power tradables is, that they allow us to construct a new basis in the space of tradables in which the matrix $A$ is 'diagonal' in the sense that every tradable depends on at most one stochastic factor. This amounts to finding a proper set of $\eta_{i}$ given a set of values for $\psi^{m}, \chi^{r}$. But this is just a linear set of equations. Indeed, taking the $\log$ of $\chi^{r}$ we see that the $\eta_{i}$ should satisfy

$$
\sum_{i=0}^{N} \eta_{i}=1, \quad \sum_{i=0}^{N} \sigma_{i}^{m} \eta_{i}=\psi^{m}, \quad \sum_{i=0}^{N} \log \left(\alpha_{i}^{r}\right) \eta_{i}=\log \left(\chi^{r}\right)
$$


This means that under obvious non-singularity conditions, it is possible to construct a new set of tradables $\mathbf{Y}$ satisfying

$$
\frac{d Y_{t}^{0}}{Y_{t_{-}}^{0}}=0, \quad \frac{d Y_{W, t}^{m}}{Y_{W, t_{-}}^{m}}=\varphi^{m} d t+d W_{t}^{m}, \quad \frac{d Y_{N, t}^{r}}{Y_{N, t_{-}}^{r}}=-\lambda^{r} d t+d N_{t}^{r}
$$

under the numeraire $Y^{0}$. This gives a very nice illustration of the concept of market price of risk under this numeraire. On the other hand, it clearly shows that the $\lambda^{r}$, and consequently the $\beta_{i}^{r}$, should be positive (almost surely) in order to avoid arbitrage in the model. Indeed, otherwise one of the $Y^{r}$ would be strictly increasing relative to $Y^{0}$, indicating an arbitrage opportunity. These no-arbitrage conditions still hold in the more general setting, but we will not explicitly derive them here. Finally note that power tradables are closely related to optimal growth or Kelly strategies. For more applications of power tradables we refer to Ref. [HN99b].

Lévy processes: the rough guide In this section we sketch how our model could be used in a simple example of an incomplete market, and, in a limit, in a market in which the price is driven by a Lévy process. We start by defining a market consisting of two tradables, a stock $S$ and a bond $P$. Taking the bond as numeraire, we assume that the stockprice is driven by one Wiener and $R$ Poisson processes. So the dynamic equations take the following form

$$
\begin{aligned}
& \frac{d S_{t}}{S_{t_{-}}}=\mu d t+\sigma d W_{t}+\sum_{r=1}^{R}\left(\alpha^{r}-1\right) d N_{t}^{r} \\
& \frac{d P_{t}}{P_{t_{-}}}=0
\end{aligned}
$$

Obviously, this market is not complete. However, we can make it complete by introducing $R$ extra tradables $P^{r}$, as follows

$$
\frac{d P_{t}^{r}}{P_{t_{-}}^{r}}=\mu_{r} d t+\left(\alpha^{r}-1\right) d N_{t}^{r}
$$

The essential freedom that we have is the choice of the drift terms $\mu_{r}$. We will only assume that they are constant, and such that the total market is arbitrage free. Now we can apply the general theory from the previous sections. Under the identification $S=X^{0}, P=X^{1}, P^{r}=X^{r+1}$ the matrix $A$ becomes

$$
A=\left(\begin{array}{cccccc}
1 & 1 & 1 & 1 & \cdots & 1 \\
\sigma & 0 & 0 & 0 & \cdots & 0 \\
\alpha^{1} & 1 & \alpha^{1} & 1 & \cdots & 1 \\
\alpha^{2} & 1 & 1 & \alpha^{2} & \cdots & 1 \\
\vdots & \vdots & \vdots & \vdots & \ddots & \vdots \\
\alpha^{R} & 1 & 1 & 1 & \cdots & \alpha^{R}
\end{array}\right)
$$

For the $\beta_{i}^{r}$ we find the following expressions

$$
\lambda_{r}=\frac{\mu_{r}}{1-\alpha^{r}}, \quad \beta_{i}^{r}= \begin{cases}\alpha^{r} \lambda_{r} & \text { if } i=0 \text { or } i=r+1 \\ \lambda_{r} & \text { otherwise }\end{cases}
$$

From this we see that the no-arbitrage conditions are simply $\mu_{r}>0$ iff $\alpha^{r}<1$ for all $r$. Now using the identity $\beta_{i}^{r}=\beta_{i}^{1}-\mu_{r}\left(\delta_{i, 0}+\delta_{i, r+1}\right)$ and homogeneity, the pricing PDDE takes the following form

$$
\begin{aligned}
0 & =\frac{\partial V(\mathbf{x}, t)}{\partial t}+\frac{1}{2} \sigma^{2} S^{2} \frac{\partial^{2} V(\mathbf{x}, t)}{\partial S^{2}} \\
& +\sum_{r=1}^{R}\left(V\left(\boldsymbol{\beta}^{r} \mathbf{x}, t\right)-V\left(\boldsymbol{\beta}^{1} \mathbf{x}, t\right)+\mu_{r} S \frac{\partial V(\mathbf{x}, t)}{\partial S}+\mu_{r} P^{r} \frac{\partial V(\mathbf{x}, t)}{\partial P^{r}}\right)
\end{aligned}
$$


Since our original market only contains $S$ and $P$, we will only be interested in contracts which are specified in terms of only these two tradables. It is not hard to see that in this case the PDDE can be reduced to

$$
\begin{aligned}
0 & =\frac{\partial V(S, P, t)}{\partial t}+\frac{1}{2} \sigma^{2} S^{2} \frac{\partial^{2} V(S, P, t)}{\partial S^{2}} \\
& +\sum_{r=1}^{R} \mu_{r}\left(S \frac{\partial V(S, P, t)}{\partial S}-\frac{V\left(\alpha^{r} S, P, t\right)-V(S, P, t)}{\alpha^{r}-1}\right)
\end{aligned}
$$

In other words, the price of such a contract will at all times remain a function of $S$ and $P$ only, and the PDDE reduces to two dimensions. It is very important to notice that this does not imply that the contract can be hedged using $S$ and $P$ only. In fact, the exact hedge ratios are given by

$$
\phi_{S}=\frac{\partial V(S, P, t)}{\partial S}, \quad \phi_{P^{r}}=\frac{V\left(\alpha^{r} S, P, t\right)-V(S, P, t)}{P^{r}\left(\alpha^{r}-1\right)}-\frac{S}{P^{r}} \frac{\partial V(S, P, t)}{\partial S}
$$

and $\phi_{P}$ follows from the portfolio replication condition. Since we can use only $S$ and $P$ in our hedge, we might consider to use a standard delta hedge $\phi_{S}=\frac{\partial V(S, P, t)}{\partial S}, \phi_{P}=\frac{\partial V(S, P, t)}{\partial P}$. In that case, we are effectively left with a position in $P$ and the virtual tradables $P^{r}$. So we should use the freedom to choose the drifts $\mu_{r}$ in such a way that we get a 'satisfactory' return on the residual risk. Obviously, this choice will depend upon the real world intensities of the Poisson processes and upon our idea of a satisfactory return.

To connect our formalism to pricing in a market driven by a Lévy process, we take a formal limit where the number of Poisson processes goes to infinity. This amounts to the replacement of the sum over $r$ by an integral over jumpsizes $\alpha$, as follows

$$
\begin{aligned}
0 & =\frac{\partial V(S, P, t)}{\partial t}+\frac{1}{2} \sigma^{2} S^{2} \frac{\partial^{2} V(S, P, t)}{\partial S^{2}} \\
& +\int_{0}^{\infty} \mu(\alpha)\left(S \frac{\partial V(S, P, t)}{\partial S}-\frac{V(\alpha S, P, t)-V(S, P, t)}{\alpha-1}\right) d \alpha \\
& \equiv\left(\partial_{t}+\mathcal{L}_{S}\right) V(S, P, t)
\end{aligned}
$$

The resulting pricing equation is a partial integro differential difference equation (PIDDE). For the case of a European type option it is possible to solve this equation. The solution will illuminate the intimate connection of our pricing theory with the theory of Lévy processess. The first step is to observe that the operators $\partial_{t}$ and $\mathcal{L}_{S}$ commute, i.e.

$$
\partial_{t}\left(\mathcal{L}_{S} V(S, P, t)\right)=\mathcal{L}_{S}\left(\partial_{t} V(S, P, t)\right)
$$

Using this fact, the solution of the pricing equation, given a payoff $V(S, P, T)$ at maturity, can be written in terms of the operator $\mathcal{L}_{S}$ as

$$
V(S, P, t)=\sum_{n=0}^{\infty} \frac{\left((T-t) \mathcal{L}_{S}\right)^{n}}{n !} V(S, P, T) \equiv e^{(T-t) \mathcal{L}_{S}} V(S, P, T)
$$

This result can be checked by direct substitution. We now concentrate on the operator $\mathcal{L}_{S}$. After a change of variable $\alpha \rightarrow e^{x}$, and introducing

$$
\psi(x) \equiv \frac{e^{x} \mu\left(e^{x}\right)}{1-e^{x}}
$$

it becomes

$$
\begin{aligned}
\mathcal{L}_{S} F(S) & =\frac{1}{2} \sigma^{2} S^{2} \frac{\partial^{2} F(S)}{\partial S^{2}} \\
& +\int_{-\infty}^{\infty} \psi(x)\left(\left(1-e^{x}\right) S \frac{\partial F(S)}{\partial S}+F\left(e^{x} S\right)-F(S)\right) d x
\end{aligned}
$$


Using partial integration ${ }^{3}$, this can be rewritten as

$$
\mathcal{L}_{S} F(S) \equiv \int_{-\infty}^{\infty}\left(\psi(x)-\lambda \delta(x)-\left(\mu-\frac{1}{2} \sigma^{2}\right) \delta^{\prime}(x)+\frac{1}{2} \sigma^{2} \delta^{\prime \prime}(x)\right) F\left(e^{x} S\right) d x
$$

where $\delta(x)$ is the Dirac-delta function and

$$
\lambda=\int_{-\infty}^{\infty} \psi(x) d x, \quad \mu=\int_{-\infty}^{\infty}\left(1-e^{x}\right) \psi(x) d x
$$

Now in order to evaluate Eq. (4.25), it will be useful to switch to momentum space. We introduce a Fourier transform operator

$$
\mathcal{F}_{x}(f)=\int_{-\infty}^{\infty} f(x) e^{i x s} d x
$$

By using the convolution theorem we obtain

$$
\mathcal{F}_{x}\left(\mathcal{L}_{S}^{n} V\left(S e^{-x}, P, T\right)\right)=\chi^{n}(s) v(s)
$$

where

$$
\begin{aligned}
\chi(s) & =\mathcal{F}_{x}\left(\psi(x)-\lambda \delta(x)-\left(\mu-\frac{1}{2} \sigma^{2}\right) \delta^{\prime}(x)+\frac{1}{2} \sigma^{2} \delta^{\prime \prime}(x)\right) \\
& =-\frac{1}{2} \sigma^{2}\left(s^{2}+i s\right)+\int_{-\infty}^{\infty}\left(e^{i s x}-1-i s\left(e^{x}-1\right)\right) \psi(x) d x
\end{aligned}
$$

and

$$
v(s)=\mathcal{F}_{x}\left(V\left(S e^{-x}, P, T\right)\right)
$$

In terms of these functions, the fourier transform of the solution becomes

$$
\mathcal{F}_{x}\left(V\left(S e^{-x}, P, t\right)\right)=e^{(T-t) \chi(s)} v(s)
$$

or, applying the convolution theorem once more

$$
V(S, P, t)=\int_{-\infty}^{\infty} p(x) V\left(S e^{x}, P, T\right) d x
$$

where

$$
\mathcal{F}_{x}(p)=e^{(T-t) \chi(s)}
$$

This formula has an obvious interpretation: the value of the option is given by the expectation of $V\left(S e^{x}, P, T\right)$, where the stochastic variable $x$ has a distribution $p(x)$ defined by the characteristic function $\exp ((T-t) \chi(s))$. The connection with the theory of infinitely divisible distributions now becomes clear [FE66]. Indeed, the measure

$$
\psi(x) d x=\frac{\mu(\alpha) d \alpha}{1-\alpha}
$$

is the canonical Lévy measure, which describes the pure jump part of the distribution $p(x)$. A very important observation is that the centering function $-i s\left(e^{x}-1\right)$ appearing in $\chi(s)$ has a non-standard

\footnotetext{
${ }^{3}$ Without going in details we mention that this operation might not be well defined, and it might be neccessary to introduce a regulating function to fully justify our results.
} 
form. This form directly follows from the pricing equation, and therefore from the fundamental principles of constructing a self-financing replicating portfolio in terms of tradables. It ensures that $\mathcal{L}_{S} S=S \chi(-i)=0$, so that $S$ itself solves the pricing equation, as it should. This is in contrast with the standard approach which starts with a Lévy process and then finds an equivalent martingale measure to price options. Furthermore, our approach gives a clear relation between the Lévy measure and the returns $\mu(\alpha)$ of the virtual tradables that we use to complete the market. The general theory gives the following constraints on the Lévy measure

$$
\int_{-\infty}^{\infty} \min \left(1, x^{2}\right) \psi(x) d x<\infty
$$

and, in order to allow the use of the non-standard centering function

$$
\int_{1}^{\infty} e^{x} \psi(x) d x<\infty
$$

In the literature, there are several examples of pricing models in which the underlying stochastic model is driven by a Lévy process. The advantage of such models is that they can in principle account for a number of stylized facts such as volatility skews and smiles and the phenomenon of sticky-volatility. One of the oldest is Merton's jump-diffusion model [Mer76]. In his model, there is a Brownian component, i.e. $\sigma>0$, and the jump part is described by the Lévy measure

$$
\psi(x)=C \exp \left(-\frac{(x-\mu)^{2}}{2 \delta^{2}}\right)
$$

A very large class of models can be described by the Lévy measure

$$
\psi(x)= \begin{cases}C_{-}|x|^{-1-\alpha} \exp \left(-\lambda_{-}|x|\right) & \text { for } x<0 \\ C_{+}|x|^{-1-\alpha} \exp \left(-\lambda_{+}|x|\right) & \text { for } x>0\end{cases}
$$

Indeed, setting $\alpha=-1$ and $\sigma>0$, this becomes Kou's jump-diffusion model [Kou01]. For $\lambda_{-}=\lambda_{+}$ and $\sigma=0$ it becomes the class of truncated Lévy processes as studied in [Mat00]. Alternatively, setting $C_{+}=C_{-}$and $\sigma=0$, we arrive at a class of models introduced by Carr, Geman, Madan and Yor [CG00]. This class contains the variance gamma model as a subclass (setting $\alpha=0$ ) [MCC98]. Finally, setting $C_{+}=\lambda_{-}=\sigma=0$, we get the maximally skewed $\alpha$-stable process studied in [CW00].

Yet another general class of models was introduced by Eberlein and Prause [EP98], the Generalized hyperbolic distributions. In this case, the Lévy measure is

$$
\psi(x)= \begin{cases}\frac{e^{\beta x}}{|x|}\left(\int_{0}^{\infty} \frac{\exp \left(-|x| \sqrt{2 y+\alpha^{2}}\right)}{\pi^{2} y\left(J_{\lambda}^{2}(\delta \sqrt{2 y})+Y_{\lambda}^{2}(\delta \sqrt{2 y})\right)} d y+\lambda \exp (-|x| \alpha)\right) & \text { for } \lambda \geq 0 \\ \frac{e^{\beta x}}{|x|} \int_{0}^{\infty} \frac{\exp \left(-|x| \sqrt{2 y+\alpha^{2}}\right)}{\pi^{2} y\left(J_{-\lambda}^{2}(\delta \sqrt{2 y})+Y_{-\lambda}^{2}(\delta \sqrt{2 y})\right)} d y & \text { for } \lambda<0\end{cases}
$$

This class contains as subclasses the hyperbolic distributions [EK95] by setting $\lambda=1$ and the normal inverse Gaussian distributions [BN97] by setting $\lambda=-1 / 2$. In the latter case, the Lévy measure simplifies to

$$
\psi(x)=\frac{e^{\beta x} \alpha \delta}{\pi|x|} K_{1}(\alpha|x|)
$$

In many of these models, it is possible to find (semi-)analytical expressions for the corresponding infinitely divisible distributions, and for European option prices. Of course, for more general types of options, one has to revert to the PIDDE Eq. (4.24), using numerical schemes to solve the equation. 


\section{RESTRICTING THE NUMBER OF JUMPS}

In the previous section we have considered markets driven by both Wiener and Poisson processes. Of course, the latter can make an infinite amount of jumps. For some price processes this might be a reasonable assumption. But there are also cases in which we might want to restrict the number of jumps. For example, when modeling credit risk it is natural to allow only one jump in the stochastic process that models this risk. In this section we will consider the consequences of such a restriction and how we can deal with them. We will focus on a restriction to one jump, but note that it is straightforward to generalize the discussion to any finite number of jumps.

\subsection{Credit risk, Duffie-Singleton}

A fundamental difference between a market driven by a Poisson process, which can make infinitely many jumps, and one driven by a stopped Poisson process, which can make only one jump, is that in the latter case the number of effective driving processes is not constant in time: the stopped Poisson process will no longer be effective after the jump. Let us consider a simple example, a market with a treasury bond $P^{0}$ and a corporate bond $P^{1}$. Taking the treasury bond as numeraire we assume the following dynamics before the jump

$$
\left.\begin{array}{l}
\frac{d P_{t}^{0}}{P_{t-1}^{0}}=0 \\
\frac{d P_{t}^{1}}{P_{t_{-}}^{1}}=\mu d t+(\alpha-1) d N_{t \wedge t_{d}}
\end{array}\right\} \text { for } t \leq t_{d}
$$

where $N_{t \wedge t_{d}} \equiv N_{\min \left(t, t_{d}\right)}$ is a Poisson process stopped after the first jump at time $t_{d}$, the time of default. We see that at default, the corporate bond jumps like

$$
P_{t_{d}}^{1}=\alpha P_{t_{d-}}^{1}
$$

i.e. the new value is a fraction of the old value. This is called 'recovery-of-market-value' by DuffieSingleton [DS95], and is a basic assumption of their credit risk model. It is to be contrasted to what they call 'recovery-of-treasury', which is an assumption of the Jarrow-Turnbull model [JT95], to be considered in the next section. To avoid arbitrage, we take the recovery rate $0<\alpha<1$ and the credit spread $\mu>0$. Now what happens after $t_{d}$ ? At that time we have two tradables and no source of randomness. This represents an overcomplete market, and we have to be careful not to introduce arbitrage opportunities. For example, we cannot use Eq. (5.1) for $t>t_{d}$ because the ratio $P^{1} / P^{0}$ is strictly increasing at that time. The most obvious way to deal with this situation is to simply drop one of the tradables. Indeed, after default, the corporate bond will no longer be traded, so it effectively seizes to exist. Still, it will have some value, and if the holder of the corporate bond does not receive this value immediately at the time of default, it will have to be reinvested in the remaining tradables. The most natural thing to do is to reinvest the money in the treasury bond. So we could say

$$
P_{t}^{1}=\frac{P_{t_{d}}^{1}}{P_{t_{d}}^{0}} P_{t}^{0}, \quad t>t_{d}
$$

Using this, we can formally extend the dynamics of the tradables to all $t$, and find, in the same numeraire

$$
\frac{d P_{t}^{1}}{P_{t_{-}}^{1}}=\mathbf{1}_{t \leq t_{d}}\left(\mu d t+(\alpha-1) d N_{t}\right)
$$

We now turn to the pricing problem in this market. It will be obvious that the price of a contract will depend on the information whether default has occurred or not. In fact, it is easy to incorporate path-dependence in the problem, since the path is fully specified by the time of default $t_{d}$. Now before default, the price will depend on both $P^{0}$ and $P^{1}$ and it will be written as $V_{0}\left(P^{0}, P^{1}, t\right)$. After default it is useful to write it as $V_{1}\left(P^{0}, P^{1}, t_{d}, t\right)$, but we have to keep the relation Eq. (5.2) in mind. 
The derivation of the pricing PDDE's follows the same lines as the general discussion in the previous section and will not be repeated. We find

$$
\begin{aligned}
& \frac{\partial V_{0}\left(P^{0}, P^{1}, t\right)}{\partial t}+V_{1}\left(\beta_{0} P^{0}, \beta_{1} P^{1}, t, t\right)-\sum_{i=0,1} \beta_{i} P^{i} \frac{\partial V_{0}\left(P^{0}, P^{1}, t\right)}{\partial P^{i}}=0 \\
& \frac{\partial V_{1}\left(P^{0}, P^{1}, s, t\right)}{\partial t}=0
\end{aligned}
$$

where

$$
\beta_{0}=\frac{\mu}{1-\alpha}, \quad \beta_{1}=\frac{\alpha \mu}{1-\alpha}
$$

The hedge ratios before default are given by

$$
\begin{aligned}
& \phi_{0}\left(P^{0}, P^{1}, t\right)=\frac{V_{0}\left(\beta_{1} P^{0}, \beta_{1} P^{1}, t\right)-V_{1}\left(\beta_{0} P^{0}, \beta_{1} P^{1}, t, t\right)}{P^{0}\left(\beta_{1}-\beta_{0}\right)} \\
& \phi_{1}\left(P^{0}, P^{1}, t\right)=\frac{V_{1}\left(\beta_{0} P^{0}, \beta_{1} P^{1}, t, t\right)-V_{0}\left(\beta_{0} P^{0}, \beta_{0} P^{1}, t\right)}{P^{1}\left(\beta_{1}-\beta_{0}\right)}
\end{aligned}
$$

Let us now proceed to solve the pricing equations for the case of a European security with maturity $T$. The payoff will be specified by a function $V_{0}\left(P^{0}, P^{1}, T\right)$ for the case that no default occurred before maturity and a function $V_{1}\left(P^{0}, P^{1}, t_{d}, T\right)$ in case default occurred at $t_{d} \leq T$. We now introduce $\tau \equiv T-t, \tau_{d} \equiv T-t_{d}, y \equiv P^{1} / P^{0}, V_{0}\left(P^{0}, P^{1}, t\right) \equiv P^{0} v_{0}(y, \tau), V_{1}\left(P^{0}, P^{1}, t_{d}, t\right) \equiv P^{0} v_{1}\left(y, \tau_{d}, \tau\right)$, and use homogeneity to arrive at

$$
\begin{aligned}
& \frac{\partial v_{0}(y, \tau)}{\partial \tau}+\left(\beta_{1}-\beta_{0}\right) y \frac{\partial v_{0}(y, \tau)}{\partial y}-\beta_{0}\left(v_{1}\left(\frac{\beta_{1}}{\beta_{0}} y, \tau, \tau\right)-v_{0}(y, \tau)\right)=0 \\
& \frac{\partial v_{1}(y, s, \tau)}{\partial \tau}=0
\end{aligned}
$$

The second equation leads to

$$
v_{1}(y, s, \tau)=v_{1}(y, s, 0)
$$

Inserting this in the first equation and making a change of variables, introducing $z \equiv y e^{-\left(\beta_{1}-\beta_{0}\right) \tau}$, we find

$$
\frac{\partial v_{0}(z, \tau)}{\partial \tau}+\beta_{0} v_{0}(z, \tau)=\beta_{0} v_{1}\left(\frac{\beta_{1}}{\beta_{0}} e^{\left(\beta_{1}-\beta_{0}\right) \tau} z, \tau, 0\right)
$$

We now multiply by an integrating factor, then integrate

$$
e^{\beta_{0} \tau} v_{0}(z, \tau)=v_{0}(z, 0)+\beta_{0} \int_{0}^{\tau} e^{\beta_{0} s} v_{1}\left(\frac{\beta_{1}}{\beta_{0}} e^{\left(\beta_{1}-\beta_{0}\right) s} z, s, 0\right) d s
$$

Rewriting this in terms of the original functions and coordinates we get this symmetric result

$$
\begin{aligned}
V_{0}\left(P^{0}, P^{1}, t\right) & =V_{0}\left(e^{-\beta_{0} \tau} P^{0}, e^{-\beta_{1} \tau} P^{1}, T\right) \\
& +\int_{t}^{T} V_{1}\left(\beta_{0} e^{-\beta_{0}(s-t)} P^{0}, \beta_{1} e^{-\beta_{1}(s-t)} P^{1}, s, T\right) d s
\end{aligned}
$$


5.2 Credit risk, Jarrow-Turnbull

Next, we consider a variation on the model treated above, now using the assumption of 'recovery-oftreasury'. As we mentioned earlier, this is a basic assumption of the Jarrow-Turnbull credit risk model [JT95]. We find that the pricing equations have exactly the same form as in the previous section, unifying the two approaches in one framework. Now under 'recovery-of-treasury' the corporate jumps, at the time of default, like

$$
P_{t_{d}}^{1}=\alpha P_{t_{d-}}^{0}
$$

i.e. the new value is a fraction of the value of the treasury bond. This corresponds to the following choice of dynamics

$$
\left.\begin{array}{l}
\frac{d P_{t}^{0}}{P_{t_{-}}^{0}}=0 \\
\frac{d P_{t}^{1}}{P_{t_{-}}^{1}}=\mu d t+\left(\frac{\alpha P_{t_{-}}^{0}}{P_{t_{-}}^{1}}-1\right) d N_{t \wedge t_{d}}
\end{array}\right\} \text { for } t \leq t_{d}
$$

So effectively we now have a tradable dependent jumpsize. We can now literally follow the discussion in the previous paragraph, and find that the pricing PDDE's and hedge ratios have exactly the same form except that the $\boldsymbol{\beta}$ are now dependent on the tradables as follows

$$
\beta_{0}=\frac{\mu P^{1}}{P^{1}-\alpha P^{0}}, \quad \beta_{1}=\frac{\alpha \mu P^{0}}{P^{1}-\alpha P^{0}}
$$

Note that if we assume that $\mu>0$, the model is arbitrage free iff $P^{1}>\alpha P^{0}$. This relation cannot be true at all times in the past. In fact there will be a $t_{c}$ such that $P_{t_{c}}^{1}=\alpha P_{t_{c}}^{0}$ and the model is arbitrage free only after this $t_{c}$. This is a fundamental problem with the recovery-of-treasury assumption. However, for most realistic problems, time will be in the proper range and we need not bother about the problem. Again we can solve the pricing PDDE's for the case of a path-dependent European security. Omitting the details of this calculation, we get

$$
\begin{aligned}
V_{0}\left(P^{0}, P^{1}, t\right) & =V_{0}\left(\frac{\left(\beta_{0}-\beta_{1}\right) P^{0}}{e^{\left(\beta_{0}-\beta_{1}\right) \tau} \beta_{0}-\beta_{1}}, \frac{\left(\beta_{1}-\beta_{0}\right) P^{1}}{e^{\left(\beta_{1}-\beta_{0}\right) \tau} \beta_{1}-\beta_{0}}, T\right) \\
& +\int_{t}^{T} \frac{\left(\beta_{0}-\beta_{1}\right)^{2} e^{(s+t)\left(\beta_{0}+\beta_{1}\right)}}{\left(e^{\beta_{0} s+\beta_{1} t} \beta_{0}-e^{\beta_{0} t+\beta_{1} s} \beta_{1}\right)^{2}} V_{1}\left(\beta_{0} P^{0}, \beta_{1} P^{1}, s, T\right) d s
\end{aligned}
$$

Note that if the payoff does not depend on the time of default, so that we have $V_{1}\left(P^{0}, P^{1}, s, T\right)=$ $V_{1}\left(P^{0}, P^{1}, T\right)$ for all $s$, the integral can be evaluated explicitely, and we get

$$
\begin{aligned}
V_{0}\left(P^{0}, P^{1}, t\right) & =V_{0}\left(\frac{\left(\beta_{0}-\beta_{1}\right) P^{0}}{e^{\left(\beta_{0}-\beta_{1}\right) \tau} \beta_{0}-\beta_{1}}, \frac{\left(\beta_{1}-\beta_{0}\right) P^{1}}{e^{\left(\beta_{1}-\beta_{0}\right) \tau} \beta_{1}-\beta_{0}}, T\right) \\
& +V_{1}\left(\frac{\beta_{0}\left(e^{\beta_{0} \tau}-e^{\beta_{1} \tau}\right) P^{0}}{\beta_{0} e^{\beta_{0} \tau}-\beta_{1} e^{\beta_{1} \tau}}, \frac{\beta_{1}\left(e^{\beta_{1} \tau}-e^{\beta_{0} \tau}\right) P^{1}}{\beta_{1} e^{\beta_{1} \tau}-\beta_{0} e^{\beta_{0} \tau}}, T\right)
\end{aligned}
$$

This simplification is what makes the recovery-of-treasury assumption attractive for path-independent options. However, for path-dependent options, the recovery-of-market-value assumption usually leads to more simple results, as we will see in the next section.

\subsection{A credit default swap}

Let us consider as an application a credit default swap (CDS) [Tav98]. This contract can be viewed as a default insurance on a corporate bond. The buyer of protection receives the difference in value before and after default in case the issuer of the corporate bond defaults during the lifetime of the CDS. In return, he has to pay the seller of protection an annuity premium until the time of default, 
or the maturity of the CDS, whichever comes first. The contract can symbolically be decomposed in more elementary contracts, each corresponding to a single cashflow, as follows

$$
\mathrm{CDS}=\text { Default }-\sum_{i} c_{i} \operatorname{Premium}\left(t_{i}\right)
$$

Here 'Default' corresponds to the insurance payoff in case of default, while the cashflow 'Premium $\left(t_{i}\right)$ ' corresponds to paying a premium of one unit of $P^{0}$ at time $t_{i}$ iff no default occurs before $t_{i}$. The $c_{i}$ are usually chosen in such a way that the initial value of the contract is zero. Now let us assume that the treasury bond, the corporate bond and the CDS all mature at the same time $T$, and (without loss of generality) that the treasury and the corporate bond have the same face value. This means that before default we have the relation

$$
P_{t}^{1}=e^{-\mu(T-t)} P_{t}^{0}
$$

Using this, the payoff of 'Default' can be described as

$$
\begin{aligned}
V_{0}\left(P^{0}, P^{1}, T\right) & =0 \\
V_{1}\left(P^{0}, P^{1}, t_{d}, T\right) & =e^{-\mu\left(T-t_{d}\right)} P^{0}-P^{1}
\end{aligned}
$$

The boundary conditions for 'Premium $\left(t_{i}\right)$ ' are given by

$$
\begin{aligned}
V_{0}\left(P^{0}, P^{1}, T\right) & =P^{0} \\
V_{1}\left(P^{0}, P^{1}, t_{d}, T\right) & =\mathbf{1}_{t_{i}<t_{d}} P^{0}
\end{aligned}
$$

Duffie-Singleton It is now straightforward to find the values of the constituent parts of the CDS in the Duffie-Singleton model before default by plugging the corresponding payoffs in Eq. (5.5), and simplifying the result using Eq. (5.7). We get

$$
\begin{aligned}
\operatorname{Default}\left(P^{0}, P^{1}, t\right) & =\left(1-e^{-\beta_{1}(T-t)}\right) \frac{\beta_{0}-\beta_{1}}{\beta_{1}} P^{1} \\
\operatorname{Premium}\left(t_{i}\right)\left(P^{0}, P^{1}, t\right) & = \begin{cases}e^{-\beta_{0}\left(t_{i}-t\right)} P^{0} & \text { if } t<t_{i} \\
P^{0} & \text { otherwise }\end{cases}
\end{aligned}
$$

Hedge ratios follow from Eqs. (5.3,5.4), taking $V_{1}\left(\beta_{0} P^{0}, \beta_{1} P^{1}, t, t\right)$ equal to $\left(\beta_{0}-\beta_{1}\right) P^{1}$ for the 'Default' contract and equal to $\mathbf{1}_{t_{i}<t} \beta_{0} P^{0}$ for 'Premium $\left(t_{i}\right)$ '.

Jarrow-Turnbull And similarly for the Jarrow-Turnbull model, using Eq. (5.6)

$$
\begin{aligned}
\operatorname{Default}\left(P^{0}, P^{1}, t\right) & =\ln \left(\frac{\beta_{1}-\beta_{0}}{\beta_{1}-e^{-\left(\beta_{1}-\beta_{0}\right)(T-t)} \beta_{0}}\right) \frac{\beta_{1}-\beta_{0}}{\beta_{0}} P^{1} \\
\operatorname{Premium}\left(t_{i}\right)\left(P^{0}, P^{1}, t\right) & = \begin{cases}\frac{\beta_{1}-\beta_{0}}{\beta_{1}-e^{-\left(\beta_{1}-\beta_{0}\right)\left(t_{i}-t\right)} \beta_{0}} P^{0} & \text { if } t<t_{i} \\
P^{0} & \text { otherwise }\end{cases}
\end{aligned}
$$

\subsection{First-to-default insurance}

In this section we consider the problem of pricing a first-to-default insurance contract on a set of $R$ corporate bonds. This contract pays the loss on the first bond to default. If none of the bonds defaults during the lifetime of the contract it expires worthless [Tav98]. We restrict our attention to corporate bonds under the recovery-of-market-value convention. We assume the following dynamics taking the treasury bond $P^{0}$ as numeraire (with $i=1, \ldots, r$ )

$$
\frac{d P_{t}^{i}}{P_{t_{-}}^{i}}=\mu_{i} d t+\sum_{r=1}^{R}\left(\alpha_{i}^{r}-1\right) d N_{t \wedge t_{d}^{r}}^{r}, \quad \text { for } t \leq \min \left(t_{d}^{r}\right)
$$


where $t_{d}^{r}$ is the time of default of $P^{r}$. A few remarks are in place. It is assumed that the process $N^{r}$ models the default process of corporate bond $P^{r}$, so that if $N^{r}$ jumps, $P^{r}$ seizes to exist. However, we do allow $N^{r}$ to influence the prices of the other corporate bonds via the elements $\alpha_{i}^{r}$ with $i \neq r$, thus introducing a form of default correlation. Because of the simple form of the contract, we will not need to know the dynamics of the bonds after any default. Now the price function $V_{0}(\mathbf{x}, t)$ before occurrence of any default should satisfy

$$
\frac{\partial V_{0}(\mathbf{x}, t)}{\partial t}+\sum_{r=1}^{R}\left(V_{r}\left(\boldsymbol{\beta}^{r} \mathbf{x}, t\right)-\sum_{i=0}^{N} \beta_{i}^{r} x_{i} \frac{\partial V_{0}(\mathbf{x}, t)}{\partial x_{i}}\right)=0
$$

where $V_{r}(\mathbf{x}, t)$ is the price of the contract given that corporate bond $P^{r}$ has defaulted. For a general contract these functions could themselves be complex derivatives on the remaining tradables. In the present case they are very simple indeed

$$
V_{r}(\mathbf{x}, t)=\left(\frac{1}{\alpha^{r}}-1\right) P^{r}
$$

Inserting this in Eq. (5.10) we are left with (since $\beta_{r}^{r}=\alpha^{r} \beta_{0}^{r}$ )

$$
\frac{\partial V_{0}(\mathbf{x}, t)}{\partial t}+\sum_{r=1}^{R}\left(\left(\beta_{0}^{r}-\beta_{r}^{r}\right) P^{r}-\sum_{i=0}^{N} \beta_{i}^{r} x_{i} \frac{\partial V_{0}(\mathbf{x}, t)}{\partial x_{i}}\right)=0
$$

To find the price of our contract, we integrate this PDE using the boundary condition $V_{0}(\mathbf{x}, T)=0$. The result is

$$
V_{0}(\mathbf{x}, t)=\sum_{r=1}^{R}\left(1-e^{-\beta_{r} \tau}\right) \frac{\beta_{0}^{r}-\beta_{r}^{r}}{\beta_{r}} P^{r}, \quad \beta_{i}=\sum_{r=1}^{R} \beta_{i}^{r}
$$

It is interesting to observe that every term in this sum has the same functional form as a default insurance on a single corporate bond, as can be seen by comparing with Eq. (5.8).

\subsection{Consistent default correlation}

The first-to-default insurance contract was relatively easy to price because of the special form of its payoff. We will now consider the pricing problem for a more general type of contract. The fundamental problem to be faced is how to formulate a model for credit-risk that is self consistent, not only before occurrence of any default, but at all times, in the presence of default correlation. We start out by generalizing Eq. (5.9) as follows

$$
\frac{d P_{t}^{i}}{P_{t_{-}}^{i}}=\mu_{i}(t) d t+\sum_{r=1}^{R}\left(\alpha_{i}^{r}(t)-1\right) d N_{t \wedge t_{d}^{r}}^{r}
$$

For the sake of simplicity we assume that the credit spreads and jump sizes only depend on time and default information but not on other tradables. Since the default times $t_{d}^{r}$ are stopping times w.r.t. the filtration $\mathcal{F}_{t}$ generated by the Poisson processes, these functions cannot be general functions of the $t_{d}^{r}$, because they should be adapted to the filtration. However, they may contain $\mathcal{F}_{t}$-measurable compositions such as $f\left(t, t_{d}^{r}\right) \mathbf{1}_{\left\{t_{d}^{r} \leq t\right\}}$. Furthermore, these functions should satisfy some self-consistency conditions, which follow from the simple observation that if a given corporate bond does not default during its lifetime, it will have a definite value at maturity, independent of possible default events of other bonds. So integration of the dynamic equations of a bond $P^{i}$ up to maturity should yield the same value for any possible path for which this bond does not default. Let us illustrate this by looking 
at a simple example. We look at two corporate bonds $P^{1}$ and $P^{2}$ and a treasury bond $P^{0}$. Taking the latter as numeraire, we write the dynamics as

$$
\begin{aligned}
& \frac{d P_{t}^{1}}{P_{t_{-}}^{1}}= \begin{cases}\mu_{1}(t) d t+\sum_{r=1,2}\left(\alpha_{1}^{r}(t)-1\right) d N_{t \wedge t_{d}^{r}}^{r} & \text { for } t \leq \min \left(t_{d}^{1}, t_{d}^{2}\right) \\
\mu_{1}\left(t_{d}^{2}, t\right) d t+\left(\alpha_{1}^{1}\left(t_{d}^{2}, t\right)-1\right) d N_{t \wedge t_{d}^{1}}^{1} & \text { for } t_{d}^{2}<t \leq t_{d}^{1} \\
0 & \text { for } t_{d}^{1}<t\end{cases} \\
& \frac{d P_{t}^{2}}{P_{t_{-}}^{2}}= \begin{cases}\mu_{2}(t) d t+\sum_{r=1,2}\left(\alpha_{2}^{r}(t)-1\right) d N_{t \wedge t_{d}^{r}}^{r} & \text { for } t \leq \min \left(t_{d}^{1}, t_{d}^{2}\right) \\
\mu_{2}\left(t_{d}^{1}, t\right) d t+\left(\alpha_{2}^{2}\left(t_{d}^{1}, t\right)-1\right) d N_{t \wedge t_{d}^{2}}^{2} & \text { for } t_{d}^{1}<t \leq t_{d}^{2} \\
0 & \text { for } t_{d}^{2}<t\end{cases}
\end{aligned}
$$

Now suppose that $P^{1}$ matures at time $T$. The consistency relations for this bond then take the form (assuming $t<t_{d}^{2}$ )

$$
\begin{aligned}
P^{1}(T) & =P^{1}(t) \exp \left(\int_{t}^{T} \mu_{1}(s) d s\right) \\
& =P^{1}(t) \alpha_{1}^{2}\left(t_{d}^{2}\right) \exp \left(\int_{t}^{t_{d}^{2}} \mu_{1}(s) d s+\int_{t_{d}^{2}}^{T} \mu_{1}\left(t_{d}^{2}, s\right) d s\right)
\end{aligned}
$$

or equivalently

$$
\alpha_{1}^{2}\left(t_{d}^{2}\right)=\exp \left(\int_{t_{d}^{2}}^{T}\left(\mu_{1}(s)-\mu_{1}\left(t_{d}^{2}, s\right)\right) d s\right)
$$

and similarly for $P^{2}$

$$
\alpha_{2}^{1}\left(t_{d}^{1}\right)=\exp \left(\int_{t_{d}^{1}}^{T}\left(\mu_{2}(s)-\mu_{2}\left(t_{d}^{1}, s\right)\right) d s\right)
$$

So we see that the off-diagonal jump sizes are completely fixed by a choice of the drift functions, and also that if one bond jumps down in response to default of another, its credit spread must go up, just as one would expect. Now consider the pricing problem. Again, the price of a contract will depend on information which of the bonds has defaulted and when, and we need four functions to describe it. The price $V_{0}(\mathbf{x}, t)$ before any default should satisfy

$$
0=\frac{\partial V_{0}(\mathbf{x}, t)}{\partial t}+\sum_{r=1,2}\left(V_{r}\left(\boldsymbol{\beta}^{r}(t) \mathbf{x}, t, t\right)-\sum_{i=0}^{2} \beta_{i}^{r}(t) x_{i} \frac{\partial V_{0}(\mathbf{x}, t)}{\partial x_{i}}\right)
$$

where the $\beta_{i}^{r}(t)$ are found in the standard way from the dynamics before any default. The functions $V_{r}\left(\mathbf{x}, t_{d}^{r}, t\right)$ with $r=1,2$ correspond to the price given that only bond $P^{r}$ has defaulted at time $t_{d}^{r}$. They satisfy

$$
\begin{aligned}
& 0=\frac{\partial V_{1}\left(\mathbf{x}, t_{d}^{1}, t\right)}{\partial t}+V_{12}\left(\boldsymbol{\beta}^{2}\left(t_{d}^{1}, t\right) \mathbf{x}, t_{d}^{1}, t, t\right)-\sum_{i=0}^{2} \beta_{i}^{2}\left(t_{d}^{1}, t\right) x_{i} \frac{\partial V_{1}\left(\mathbf{x}, t_{d}^{1}, t\right)}{\partial x_{i}} \\
& 0=\frac{\partial V_{2}\left(\mathbf{x}, t_{d}^{2}, t\right)}{\partial t}+V_{12}\left(\boldsymbol{\beta}^{1}\left(t_{d}^{2}, t\right) \mathbf{x}, t, t_{d}^{2}, t\right)-\sum_{i=0}^{2} \beta_{i}^{1}\left(t_{d}^{2}, t\right) x_{i} \frac{\partial V_{2}\left(\mathbf{x}, t_{d}^{2}, t\right)}{\partial x_{i}}
\end{aligned}
$$

In this case, $\beta_{0}^{1}\left(t_{d}^{2}, t\right)$ and $\beta_{1}^{1}\left(t_{d}^{2}, t\right)$ are found from the dynamics of $P^{0}$ and $P^{1}$ given that $P^{2}$ has defaulted at time $t_{d}^{2}$ and $P^{1}$ has not. Next, $\beta_{2}^{1}\left(t_{d}^{2}, t\right)$ should be taken equal to $\beta_{0}^{1}\left(t_{d}^{2}, t\right)$, corresponding 
to the fact that $P^{2}$ has the same dynamics as the treasury. A similar story holds for the $\beta_{i}^{2}\left(t_{d}^{1}, t\right)$. Finally, the price $V_{12}\left(\mathbf{x}, t_{d}^{1}, t_{d}^{2}, t\right)$ after default of both corporate bonds simply satisfies

$$
0=\frac{\partial V_{12}\left(\mathbf{x}, t_{d}^{1}, t_{d}^{2}, t\right)}{\partial t}
$$

In general, this set of equations will be hard to solve and we will have to revert to numerical techniques. It is a challenge to find a model which is analytically managable, while still incorporating default correlation in a realistic way. We will return to this problem in future work.

\section{Conclusions and outlook}

Using local scale invariance, a.k.a. numeraire invariance, as a first fundamental principle we have shown how one can derive derivative security prices, and hedge ratios, in a completed market with underlying price processes driven by Wiener and Poisson processes. We discussed the various symmetries that should be satisfied by the dynamic equation governing the prices of derivative securities and the subtle differences regarding the notion of 'market price of risk' between the Wiener and Poisson case. We have further indicated how to extend the jump-diffusion case to Lévy processes. The complete market case provides a natural basis to introduce the effects of incomplete markets. We showed how this leads to PIDDE where a 'market-price' of risk has to be specified via some function on the jump-size. This provides a natural and intuitive starting point for generating corrections to the usual Wiener case. When applied to stock options, such models give an alternative way to explain and model the volatility surface, which seems to be in better agreement with the observed behaviour then the standard 'local volatility' approach [AA00].

Another application that bears the fruit of making the symmetries explicit is credit derivatives. In that case we were able to show how both the Duffie-Singleton and Jarrow-Turnbull approaches can be understood from one encompassing framework. Furthermore we introduced a 'market model' for credit risk and showed how to compute first-to-default insurance contracts in such a framework. The important notion of credit correlation can be modeled in a consistent and straightforward manner using our framework.

Symmetries invoke constraints on model building and as such they provide guidance in how to construct good models for derivative security prices. The general pricing equation derived for Lévy processes provides a good starting point for generating solutions in some perturbative expansion. Such approximations can be used to mark the model to the market for example.

The credit correlation model can be computed numerically for arbitrary choices of the parameters that determine the stochastic dynamics. It is however important to have a simple, easily calculable model that provides swift and accurate prices and hedge ratios for credit derivative securities.

We will come back to these points in future work.

\section{A. Time Dependent CASE}

In this appendix we will give a proof by direct substitution of the correctness of the general solution as given in Sec. 4.2. For a more direct derivation we refer to the results obtained in Ref. [HN99a] (for the diffusion part) and Sec. 4.1 (for the jump part). The PDDE to be solved is given by

$$
\begin{aligned}
0= & \frac{\partial V(\mathbf{x}, t)}{\partial t}+\frac{1}{2} \sum_{i, j=0}^{N} \sum_{m=1}^{M} \sigma_{i}^{m}(t) \sigma_{j}^{m}(t) x_{i} x_{j} \frac{\partial^{2} V(\mathbf{x}, t)}{\partial x_{i} \partial x_{j}} \\
& +\sum_{r=1}^{R}\left(V\left(\boldsymbol{\beta}^{r}(t) \mathbf{x}, t\right)-\sum_{i=0}^{N} \beta_{i}^{r}(t) x_{i} \frac{\partial V(\mathbf{x}, t)}{\partial x_{i}}\right) .
\end{aligned}
$$

where the $\beta_{i}^{r}(t)$ are defined by

$$
\beta_{i}^{r}(t) \equiv \alpha_{i}^{r} \lambda_{r}(t), \quad \lambda_{r}(t) \equiv-\sum_{j=0}^{N} \mu_{j}(t) A_{j, M+r}^{-1}(t)
$$


Note that the $\alpha_{i}^{r}$ are assumed to be constant. We will see that this assumption is crucial to get a simple form for the solution. The reason is that if jumpsizes are not constant, effects of jumps can no longer be described in terms of the number of jumps that occur, but one needs exact information on the timing of the jumps. To solve the PDDE, we use the following ansatz for the solution

$$
V(\mathbf{x}, t)=\sum_{\mathbf{n} \geq 0} \int_{\mathbf{R}^{M}} g\left(\left\{x_{i} \phi\left(\mathbf{z}-\boldsymbol{\theta}_{i}\right) \pi_{\mathbf{n}}\left(\boldsymbol{\rho}_{i}\right)\right\}\right) d \mathbf{z}
$$

Here $g(\mathbf{x})=V(\mathbf{x}, T)$ describes the boundary condition at maturity $T$. The vectors $\boldsymbol{\theta}_{i} \equiv\left(\theta_{i}^{1}, \ldots, \theta_{i}^{M}\right)$ follow from a singular value decomposition of the time-integrated covariance matrix

$$
\sum_{m=1}^{M} \theta_{i}^{m}(t) \theta_{j}^{m}(t) \equiv \int_{t}^{T} \sum_{m=1}^{M} \sigma_{i}^{m}(u) \sigma_{j}^{m}(u) d u
$$

In a similar way, the vectors $\boldsymbol{\rho}_{i} \equiv\left(\rho_{i}^{1}, \ldots, \rho_{i}^{R}\right)$ are defined as

$$
\rho_{i}^{r}(t) \equiv \int_{t}^{T} \beta_{i}^{r}(u) d u
$$

The $\phi(\mathbf{z})$ and $\pi_{\mathbf{n}}\left(\boldsymbol{\rho}_{i}\right)$ are defined by

$$
\begin{aligned}
\phi(\mathbf{z}) & \equiv \prod_{m=1}^{M} \frac{1}{\sqrt{2 \pi}} e^{-\frac{1}{2} z_{m}^{2}} \\
\pi_{\mathbf{n}}\left(\boldsymbol{\rho}_{i}\right) & \equiv \prod_{r=1}^{R} \frac{\left(\rho_{i}^{r}\right)^{n_{r}}}{n_{r} !} e^{-\rho_{i}^{r}}
\end{aligned}
$$

To prove that this ansatz is indeed the solution of the PDDE we consider the two seperate cases, where the jumps and diffusions are switched off respectively. Leibnitz' rule then suffices to prove that the case with both diffusions and jumps is solved by the ansatz Eq. (A.1). We start with the diffusion part, showing that the function

$$
V(\mathbf{x}, t)=\int_{\mathbf{R}^{M}} g\left(\left\{x_{i} \phi\left(\mathbf{z}-\boldsymbol{\theta}_{i}\right)\right\}\right) d \mathbf{z}
$$

solves the PDE

$$
0=\mathcal{L} V(\mathbf{x}, t)=\frac{\partial V(\mathbf{x}, t)}{\partial t}+\frac{1}{2} \sum_{i, j=0}^{N} \sum_{m=1}^{M} \sigma_{i}^{m}(t) \sigma_{j}^{m}(t) x_{i} x_{j} \frac{\partial^{2} V(\mathbf{x}, t)}{\partial x_{i} \partial x_{j}}
$$

To this end, we need the following identities, which are easy to derive

$$
\begin{aligned}
\frac{\partial}{\partial t}\left(\sum_{m=1}^{M} \theta_{i}^{m}(t) \theta_{j}^{m}(t)\right) & =-\sum_{m=1}^{M} \sigma_{i}^{m}(t) \sigma_{j}^{m}(t) \\
\frac{\partial \phi(\mathbf{z})}{\partial z_{m}} & =-z_{m} \phi(\mathbf{z}) \\
\frac{\partial g}{\partial t} & =\sum_{i, m}\left(z_{m}-\theta_{i}^{m}(t)\right) \frac{\partial \theta_{i}^{m}(t)}{\partial t} x_{i} \frac{\partial g}{\partial x_{i}} \\
\frac{\partial g}{\partial z_{m}} & =\sum_{i}\left(z_{m}-\theta_{i}^{m}(t)\right) x_{i} \frac{\partial g}{\partial x_{i}}
\end{aligned}
$$


From these we can derive the crucial equation

$$
\begin{aligned}
\mathcal{L} g & =\sum_{i, m}\left(z_{m}-\theta_{i}^{m}(t)\right) \frac{\partial \theta_{i}^{m}(t)}{\partial t} x_{i} \frac{\partial g}{\partial x_{i}}-\sum_{i, j, m} \theta_{i}^{m}(t) \frac{\partial \theta_{j}^{m}(t)}{\partial t} x_{i} x_{j} \frac{\partial^{2} g}{\partial x_{i} \partial x_{j}} \\
& =\sum_{i, j, m}\left(z_{m}-\theta_{i}^{m}(t)\right) \frac{\partial \theta_{j}^{m}(t)}{\partial t}\left(x_{i} \frac{\partial}{\partial x_{i}}\right)\left(x_{j} \frac{\partial}{\partial x_{j}}\right) g \\
& =\sum_{j, m} \frac{\partial \theta_{j}^{m}(t)}{\partial t}\left(x_{j} \frac{\partial}{\partial x_{j}}\right) \frac{\partial g}{\partial z_{m}}
\end{aligned}
$$

where Eqs. (A.2,A.3) were used in the first step, homogeneity in the second and Eq. (A.4) in the third. If we plug this into the PDE we find

$$
\mathcal{L} V(\mathbf{x}, t)=\int_{\mathbf{R}^{M}} \mathcal{L} g d \mathbf{z}=\sum_{j, m} \frac{\partial \theta_{j}^{m}(t)}{\partial t}\left(x_{j} \frac{\partial}{\partial x_{j}}\right) \int_{\mathbf{R}^{M}} \frac{\partial g}{\partial z_{m}} d \mathbf{z}
$$

The integral over $z_{m}$ becomes trivial and we see that the expression vanishes because of the strong decay of the Gaussian at infinity.

Next, we consider the jump part, showing that the function

$$
V(\mathbf{x}, t)=\sum_{\mathbf{n} \geq 0} g\left(\left\{x_{i} \pi_{\mathbf{n}}\left(\boldsymbol{\rho}_{i}\right)\right\}\right) \equiv \sum_{\mathbf{n} \geq 0} g_{\mathbf{n}}
$$

solves the equation

$$
0=\mathcal{L} V(\mathbf{x}, t)=\frac{\partial V(\mathbf{x}, t)}{\partial t}+\sum_{r=1}^{R}\left(V\left(\boldsymbol{\beta}^{r}(t) \mathbf{x}, t\right)-\sum_{i=0}^{N} \beta_{i}^{r}(t) x_{i} \frac{\partial V(\mathbf{x}, t)}{\partial x_{i}}\right)
$$

We first derive some useful identities

$$
\begin{aligned}
\frac{\partial \pi_{\mathbf{n}}\left(\boldsymbol{\rho}_{i}\right)}{\partial t} & =\sum_{r=1}^{R}\left(\pi_{\mathbf{n}}\left(\boldsymbol{\rho}_{i}\right)-\pi_{\mathbf{n}-\delta_{r}}\left(\boldsymbol{\rho}_{i}\right) \mathbf{1}_{n_{r} \geq 1}\right) \beta_{i}^{r}(t) \\
\pi_{n}\left(\boldsymbol{\rho}_{i}\right) \beta_{i}^{r}(t) & =\pi_{\mathbf{n}+\delta_{r}}\left(\boldsymbol{\rho}_{i}\right) \frac{\beta_{i}^{r}(t)}{\rho_{i}^{r}(t)}\left(n_{r}+1\right)
\end{aligned}
$$

where $\delta_{r}$ is a vector with a one at position $r$ and zeros elsewhere. The important thing to note in the last equation is that the ratio of $\beta_{i}^{r}$ and $\rho_{i}^{r}$ does not depend on the jumpsize $\alpha_{i}^{r}$ by virtue of the fact that the $\alpha_{i}^{r}$ are constants, as can be easily seen from the definition of the $\beta_{i}^{r}$ 's:

$$
\frac{\beta_{i}^{r}(t)}{\rho_{i}^{r}(t)}=\frac{\lambda^{r}(t)}{\int_{t}^{T} \lambda^{r}(u) d u}
$$

Therefore it does not depend on the index $i$ and this allows us to exploit homogeneity again. Indeed, using the last equation and the fact that $\partial_{i} g(\mathbf{x})$ is homogeneous of degree zero, making it possible to scale out an arbitrary overall factor, we can write

$$
\partial_{i} g\left(\left\{x_{j} \pi_{\mathbf{n}}\left(\boldsymbol{\rho}_{j}\right) \beta_{j}^{r}(t)\right\}\right)=\partial_{i} g_{\mathbf{n}+\delta_{r}}
$$

where $\partial_{i}$ denotes the derivative w.r.t. the $i$-th argument. Since $g(\mathbf{x})$ is homogeneous of degree 1 , $\partial_{i} g(\mathbf{x})$ is homogeneous of degree 0 and we can scale out an arbitrary overall factor. By Eq. (A.5) we 
find

$$
\begin{aligned}
\frac{\partial g_{\mathbf{n}}}{\partial t} & =\sum_{i=0}^{N} \frac{\partial \pi_{\mathbf{n}}\left(\boldsymbol{\rho}_{i}\right)}{\partial t} x_{i} \partial_{i} g_{\mathbf{n}} \\
& =\sum_{r=1}^{R} \sum_{i=0}^{N}\left(\pi_{\mathbf{n}}\left(\boldsymbol{\rho}_{i}\right)-\pi_{\mathbf{n}-\delta_{r}}\left(\boldsymbol{\rho}_{i}\right) \mathbf{1}_{n_{r} \geq 1}\right) \beta_{i}^{r}(t) x_{i} \partial_{i} g_{\mathbf{n}}
\end{aligned}
$$

Homogeneity together with Eq. (A.6) leads to

$$
\begin{aligned}
\sum_{r=1}^{R} g\left(\left\{x_{j} \pi_{\mathbf{n}}\left(\boldsymbol{\rho}_{j}\right) \beta_{j}^{r}(t)\right\}\right) & =\sum_{r=1}^{R} \sum_{i=0}^{N} \pi_{\mathbf{n}}\left(\boldsymbol{\rho}_{i}\right) \beta_{i}^{r}(t) x_{i} \partial_{i} g\left(\left\{x_{j} \pi_{\mathbf{n}}\left(\boldsymbol{\rho}_{j}\right) \beta_{j}^{r}(t)\right\}\right) \\
& =\sum_{r=1}^{R} \sum_{i=0}^{N} \pi_{\mathbf{n}}\left(\boldsymbol{\rho}_{i}\right) \beta_{i}^{r}(t) x_{i} \partial_{i} g_{\mathbf{n}+\delta_{r}}
\end{aligned}
$$

Finally

$$
-\sum_{r=1}^{R} \sum_{i=0}^{N} \beta_{i}^{r}(t) x_{i} \frac{\partial g_{\mathbf{n}}}{\partial x_{i}}=-\sum_{r=1}^{R} \sum_{i=0}^{N} \pi_{\mathbf{n}}\left(\boldsymbol{\rho}_{i}\right) \beta_{i}^{r}(t) x_{i} \partial_{i} g_{\mathbf{n}}
$$

Putting this al together, we find after some renumbering

$$
\mathcal{L} V(\mathbf{x}, t)=\sum_{\mathbf{n} \geq 0} \mathcal{L} g_{\mathbf{n}}=0
$$

and this concludes the proof. 


\section{References}

[AA00] L. Andersen and J. Andreasen, Jump-Diffusion processes: Volatility Smile Fitting and Numerical Methods for Pricing, Working paper, General Re Financial Products, (2000).

[BN97] O.E. Barndorff-Nielsen, Normal Inverse Gaussian Distributions and Stochastic Volatility Modelling, Scandinavian Journal of Statistics 24, (1997), 1-13.

[BS73] F. Black and M. Scholes, The Pricing of Options and Corporate Liabilities, Journal of Political Economy, 81, (1973), 637:659.

[BH99] J.G.B. Beumee, B. Hilberink, S. Patel, and P. Walsh, Hedging Derivative Credit Risk, Derivative Credit Risk (2nd Edition), Risk Books, (1999).

[CG00] P. Carr, H. Geman, D. Madan and M. Yor, The fine structure of asset returns: An emperical investigation, Working paper, (2000).

[CW00] P. Carr and L. Wu, The Finite Moment Logstable Process and Option Pricing, Working paper, (2000).

[DS95] D. Duffie and K. Singleton, Recursive Valuation of Defaultable Securities and the timing of Resolution of Uncertainty, Working Paper, Stanford University, (1995).

[EK95] E. Eberlein and U. Keller, Hyperbolic Distributions in Finance, Bernoulli 1, (1995), 281-299.

[EP98] E. Eberlein and K. Prause, The Generalized Hyperbolic Model: Financial Derivatives and Risk Measures, FDM preprint 56, University of Freiburg, (1998).

[FE66] W. Feller, An introduction to probability theory and applications, Vol. II, Wiley, New York (1966).

[GKR95] H. Geman, N. El Karoui and J.C. Rochet, Changes of numeraire, changes of probability measure and option pricing, J.Appl.Probab. 32, (1995), 2, 443-458.

[HP81] J.M. Harrison and S. Pliska, Martingales and Stochastic Integrals in the Theory of Continuous Trading, Stoch.Process.Appl. 11, (1981), 215:260.

[HN99a] J.K. Hoogland and C.D.D. Neumann, Local Scale Invariance and Contingent Claim Pricing I, IJTAF, 4, (2001), 1, 1-21.

[HN99b] J.K. Hoogland and C.D.D. Neumann, Local Scale Invariance and Contingent Claim Pricing II: Path-dependent Contingent Claims, IJTAF, 4, (2001), 1, 23-43.

[HN00a] J.K. Hoogland and C.D.D. Neumann, Asians and Cash Dividends: Exploiting Symmetries in Pricing Theory, Technical Report MAS-R0019, CWI, (2000). 
[HN00b] J.K. Hoogland and C.D.D. Neumann, Tradable Schemes, Technical Report MAS-R0024, CWI, (2000).

[HN00c] J.K. Hoogland and C.D.D. Neumann, Local Scale Invariance in Option Pricing, Proceedings CRM 2000, Eds. L. Seco and S. Tompaidis, (2000).

[JM95] R.A. Jarrow and D. Madan, Option Pricing Using the Term Structure of Interest Rates to Hedge Systematic Discontinuities in Asset Returns, Mathematical Finance, 5, (1995), 4, 311-336.

[JT95] R.A. Jarrow and S.M. Turnbull, Pricing Derivatives on Financial Securities Subject to Credit Risk, Journal of Finance, 1, (1995), 53-85.

[Jen99] B. Jensen, Option Pricing in the Jump-diffusion Model with a Random Jump Amplitude: A Complete Market Approach, Working paper no.42, Center for Analytical Finance, University of Aarhus, (1999).

[Kou01] S.G. Kou, A Jump Diffusion Model for Option Pricing, Working paper, Columbia University, (2001).

[MCC98] D. Madan, P. Carr and E. Chang, The Variance Gamma Process and Option Pricing, European Finance Review 2, (1998), 79-105.

[Mat00] A. Matacz, Financial Modeling and Option Pricing Theory with the Truncated Lévy Process, IJTAF, 3, (2000), 1, 143-160.

[Mer76] R.C. Merton, Option Pricing when Underlying Stock Returns are Discontinuous, Journal of Financial Economics, 3, (1976), 125-144.

[Tav98] J.M. Tavakoli, Credit Derivatives, A Guide to Instruments and Applications, John Wiley \& Sons, (1998).

[VBH99] M.H. Vellekoop, J.G. Beumee and Hilberink, Pricing and Hedging Options on Defaultable Assets: An Explicit Tree Method, Working paper, (1999). 